OECD Series on Adverse Outcome Pathways No. 1

\title{
Users' Handbook
} supplement to the Guidance

Document for developing OECD and assessing Adverse Outcome Pathways 
ENVIRONMENT DIRECTORATE

JOINT MEETING OF THE CHEMICALS COMMITTEE AND THE WORKING PARTY ON CHEMICALS, PESTICIDES AND BIOTECHNOLOGY

Cancels \& replaces the same document of 27 January 2017

\section{USERS' HANDBOOK SUPPLEMENT TO THE GUIDANCE DOCUMENT FOR DEVELOPING AND ASSESSING AOPS}

Series on Testing \& Assessment

No. 233

Series on Adverse Outcome Pathways

No. 1

Second Edition of the Users' Handbook, replacing the version dated 2017.

JT03426727 
2 ENV/JM/MONO(2016)12 
OECD Environment, Health and Safety Publications

Series on Testing and Assessment

No. 233

USERS' HANDBOOK SUPPLEMENT TO THE GUIDANCE DOCUMENT FOR DEVELOPING AND ASSESSING AOPs

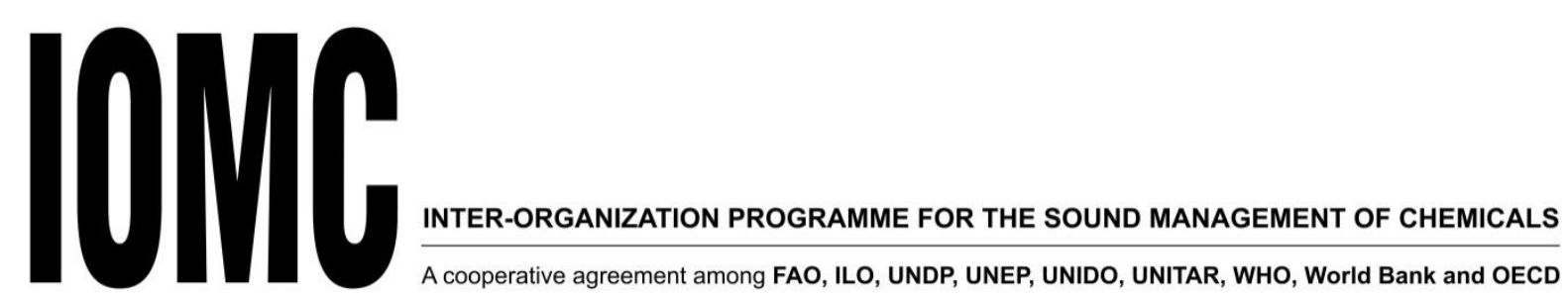

Environment Directorate ORGANISATION FOR ECONOMIC CO-OPERATION AND DEVELOPMENT Paris 2016 


\begin{abstract}
About the OECD
The Organisation for Economic Co-operation and Development (OECD) is an intergovernmental organisation in which representatives of 35 industrialised countries in North and South America, Europe and the Asia and Pacific region, as well as the European Commission, meet to co-ordinate and harmonise policies, discuss issues of mutual concern, and work together to respond to international problems. Most of the OECD's work is carried out by more than 200 specialised committees and working groups composed of member country delegates. Observers from several countries with special status at the OECD, and from interested international organisations, attend many of the OECD's workshops and other meetings. Committees and working groups are served by the OECD Secretariat, located in Paris, France, which is organised into directorates and divisions.

The Environment, Health and Safety Division publishes free-of-charge documents in twelve different series: Testing and Assessment; Good Laboratory Practice and Compliance Monitoring; Pesticides; Biocides; Risk Management; Harmonisation of Regulatory Oversight in Biotechnology; Safety of Novel Foods and Feeds; Chemical Accidents; Pollutant Release and Transfer Registers; Emission Scenario Documents; Safety of Manufactured Nanomaterials; and Adverse Outcome Pathways. More information about the Environment, Health and Safety Programme and EHS publications is available on the OECD's World Wide Web site (www.oecd.org/chemicalsafety/).
\end{abstract}

This publication was developed in the IOMC context. The contents do not necessarily reflect the views or stated policies of individual IOMC Participating Organizations.

The Inter-Organisation Programme for the Sound Management of Chemicals (IOMC) was established in 1995 following recommendations made by the 1992 UN Conference on Environment and Development to strengthen co-operation and increase international co-ordination in the field of chemical safety. The Participating Organisations are FAO, ILO, UNDP, UNEP, UNIDO, UNITAR, WHO, World Bank and OECD. The purpose of the IOMC is to promote co-ordination of the policies and activities pursued by the Participating Organisations, jointly or separately, to achieve the sound management of chemicals in relation to human health and the environment. 
This publication is available electronically, at no charge.

For this and many other Environment, Health and Safety publications, consult the OECD's World Wide Web site (www.oecd.org/ehs)

or contact:

OECD Environment Directorate, Environment, Health and Safety Division

2, rue André-Pascal

75775 Paris cedex 16

France

Fax : (33-1) 44306180

E-mail : ehscont@oecd.org

C OECD 2016

Applications for permission to reproduce or translate all or part of this material should be made to: Head of Publications Service, RIGHTS@oecd.org, OECD, 2 rue André-Pascal, 75775 Paris Cedex 16, France 


\section{Foreword}

This document is the Users' Handbook supplement to the Guidance Document for developing and assessing Adverse Outcome Pathways (AOPs) [ENV/JM/MONO(2013)6, Second Edition]. The latter provides a historical background for the AOP development programme, and outlines the elements required to construct an AOP as well as the principles of the AOP framework.

The Users' Handbook supplement was prepared initially in June 2014 by a subgroup of the OECD's Extended Advisory Group on Molecular Screening and Toxicogenomics (EAGMST). At that time it was acknowledged that the Handbook should be revised once expert groups and member countries acquire experience in developing and assessing AOPs. Since then, some experience has been gained in developing AOPs, and the material related to the AOP knowledgebase (AOP-KB) and the weight of evidence (WoE) considerations has significantly evolved. For these reasons, the Handbook was revised by the same subgroup of EAGMST to amend and incorporate new material based on lessons learned.

The Users' Handbook was reviewed and discussed by EAGMST at the 10th meeting of the EAGMST, in June 2017, and endorsed by EAGMST through written procedure in July 2017. It was subsequently sent to the Working Group of the National Coordinators of the Test Guidelines Programme and to the Working Party on Hazard Assessment who approved it by written procedure.

The Joint Meeting of the Chemicals Committee and the Working Party on Chemicals, Pesticides and Biotechnology agreed to declassification of this document in January 2018

This document is being published under the responsibility of the Joint Meeting of the Chemicals Committee and the Working Party on Chemicals, Pesticides and Biotechnology. 


\section{Table of contents}

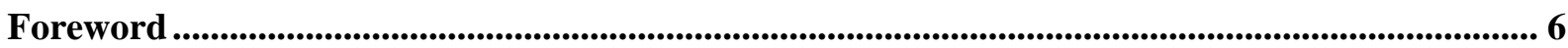

Executive Summary .............................................................................................................................................. 9

Introduction to Adverse Outcome Pathways (AOPs) ......................................................................... 12

Obtaining Author Access to the AOP-KB ............................................................................................... 16

A Note on AOP Descriptions in the AOP-KB .......................................................................................... 17

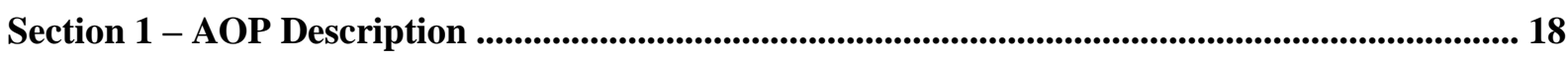

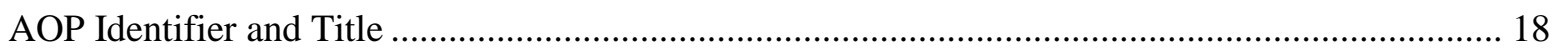

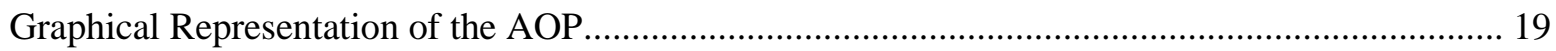

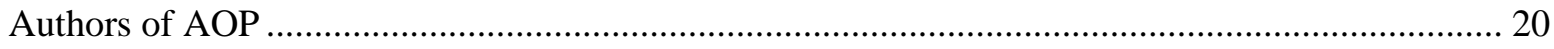

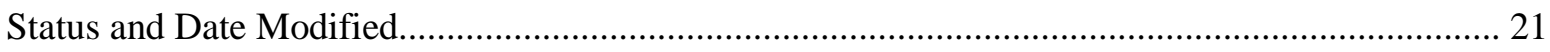

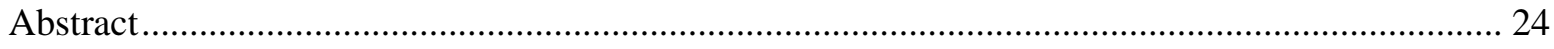

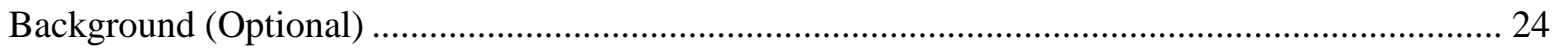

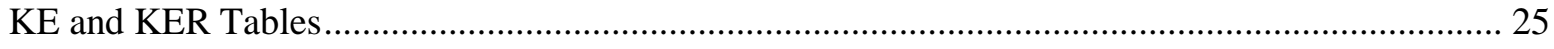

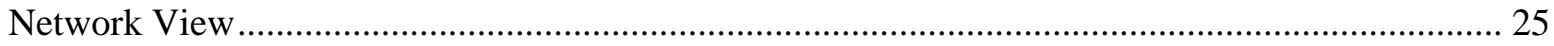

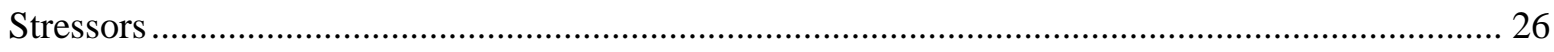

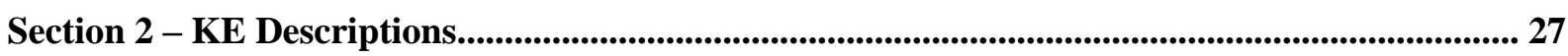

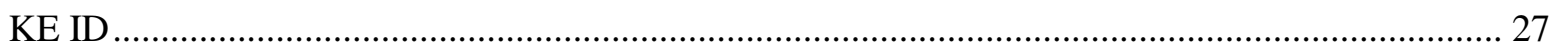

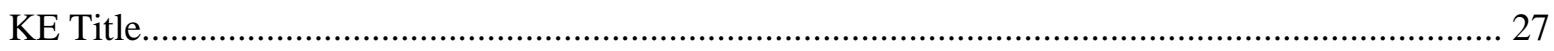

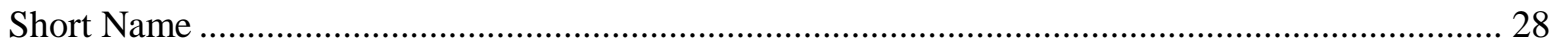

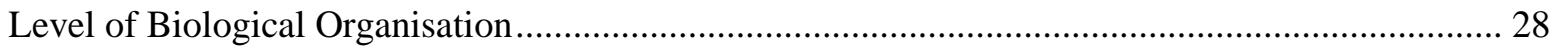

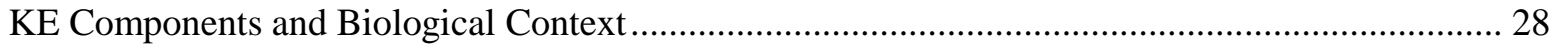

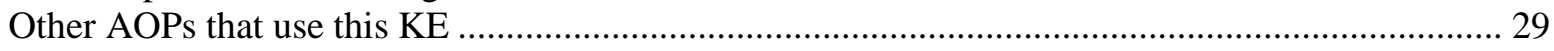

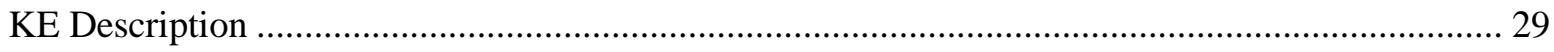

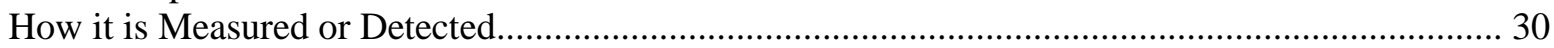

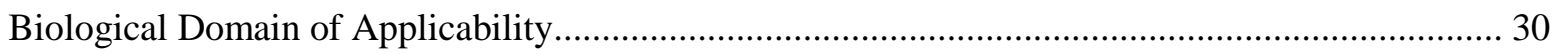

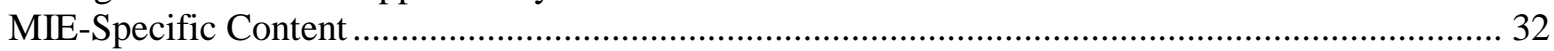

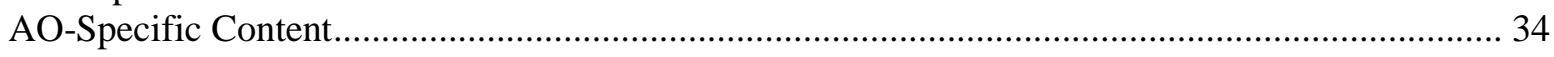

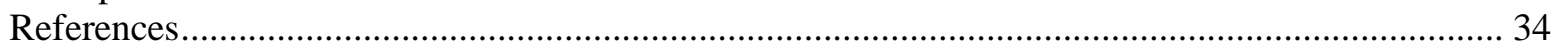

Section 3 - KER Descriptions......................................................................................................................... 35

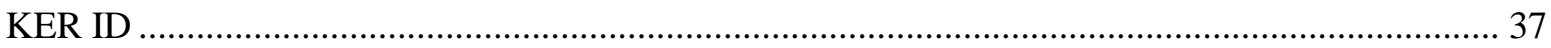

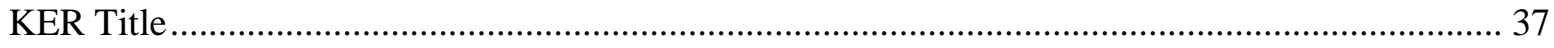

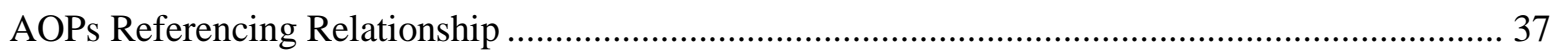

Biological Domain of Applicability.................................................................................... 37

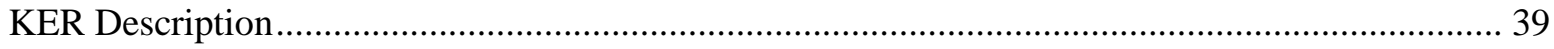

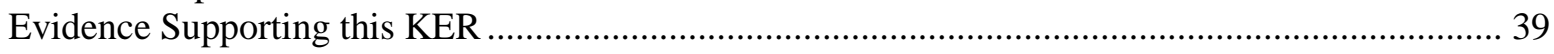

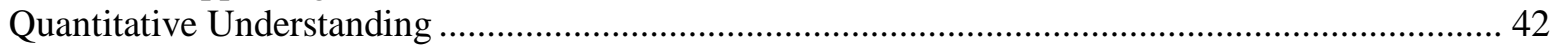




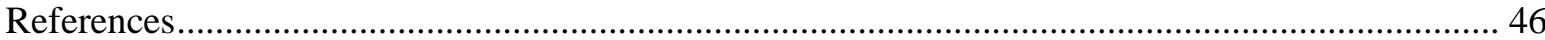

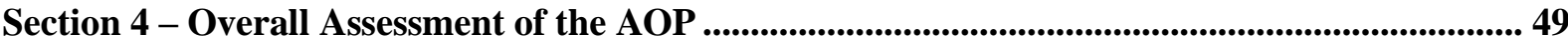

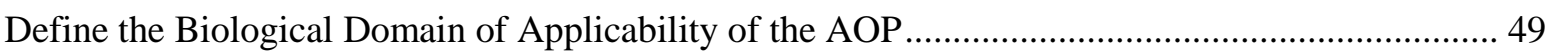

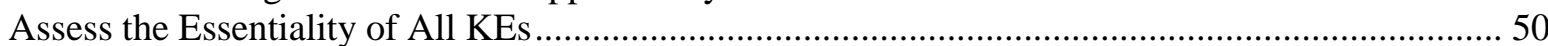

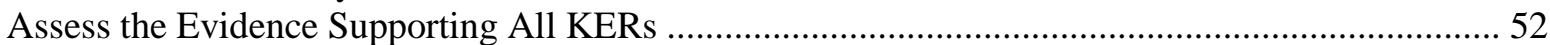

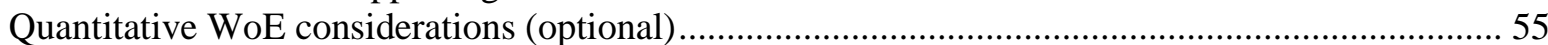

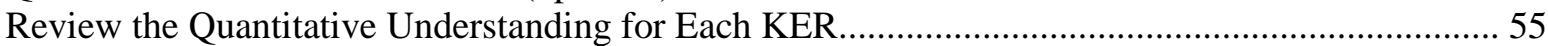

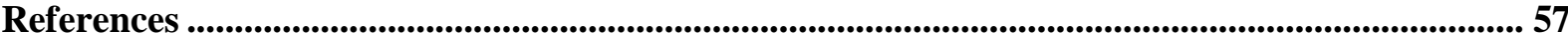

Annex 1: Guidance for Assessing Relative Level of Confidence in the Overall AOP ................... 59

Annex 2: General guidance for characterising the level of quantitative understanding of a KER as low, moderate, or high............................................................................................................................................. 62 


\section{Executive Summary}

This document is a supplement to the Guidance Document for developing and assessing Adverse Outcome Pathways (AOPs) [ENV/JM/MONO(2013)6] (AOP Guidance hereafter).

The AOP Guidance, originally published in 2013 and revised in 2017, provides an introduction to the terminology and concepts of AOP development, including the identification and use of relevant scientific data and resulting knowledge. The Guidance also briefly outlines some potential applications of AOPs.

While the AOP Guidance provides a set of definitions and the conceptual background behind AOP development, the present document, the OECD AOP Users' Handbook, is designed to provide focused, in-depth, and practical instructions concerning development and review of AOP-descriptions disseminated through the internationally harmonised AOP knowledgebase (AOP-KB, http://aopkb.org or https://aopkb.oecd.org/). The Users' Handbook can be thought of as being analogous to the "instructions for authors" used in preparing a journal article. However, in this case, rather than describing the preparation of a technical manuscript, this Handbook details how to structure an AOP description in the AOP-KB. This handbook contains an updated template for AOP development that is organised into sections. Each section of the handbook described below aligns with corresponding sections within the pages to be constructed within the AOP-KB (Table 1). In this manner, the Handbook is intended to assist in identifying, organising and evaluating critical information on key events (KEs) as well as linkages between KEs, termed key event relationships (KERs), within the AOP (i.e., AOP development). It also provides more explicit guidance on how to assemble and assess the weight of evidence (WoE) (degree of confidence) supporting the AOP and its relevance for different life stages, sex, taxa, etc. The content of the Handbook is specifically designed to support entry of AOP information into the AOP-Wiki (http://aopwiki.org, one module of the AOP-KB), and will be updated and harmonised as the AOP-KB evolves.

As with the AOP Guidance itself, this Handbook is not intended to provide a review or summary of the literature informing the AOP concept. Instead, it focuses on practical aspects of AOP development and assessment. Likewise, the Handbook is not intended to provide guidance on determining the appropriate or inappropriate regulatory application of AOPs. However, by following the template and practices outlined in the Users' Handbook, AOP developers should be in a position to systematically and efficiently assemble information pertinent to their AOP (the focus of Sections 1-3), and evaluate the underlying WoE (the focus of Section 4). This should provide transparent assessment of the level of confidence in the overall AOP, as well as critical gaps and uncertainties that 
are relevant to decisions regarding appropriate regulatory applications. Although potential regulatory applications (e.g., developing Test Guidelines, forming categories, informing integrated approaches to testing and assessment (IATA), or risk assessments within different regulatory contexts) may be described in Section 4, this information is considered optional.

AOP descriptions developed as part of the OECD AOP Development Programme are peer-reviewed as per procedures outlined by the OECD. Because AOP descriptions within the AOP-KB are viewed as living documents, they are expected to continue to evolve over time as new evidence supporting or rejecting AOPs are generated and/or new knowledge is gained. Consequently, AOPs that are reviewed and endorsed by the OECD will have multiple versions, namely, the version that existed at the time of the review and endorsement and the current version that exists in the AOP-KB. Reviews are performed on "snapshots" of content from the AOP-KB, as it existed when review was initiated. These snapshots are permanently stored in the AOP-KB along with the living document to clearly distinguish between the version of the AOP that has been endorsed and the current state of knowledge. The snapshot corresponding to the endorsed version of the AOP are also published in the OECD series on Adverse Outcome Pathways [http://www.oecd-ilibrary.org/environment/oecd-series-on-adverse-outcome-

pathways_2415170x]. The AOP-KB allows the download of both current AOP information and all snapshots in PDF form. It also provides tools for examining the differences between any snapshot and the current version of the AOP.

The sections in the handbook are presented in the order in which information should be assembled during AOP development. Based on feedback from previous AOP reviewers, it was determined that this is not optimal for AOP review. As a result, while the "snapshots" extracted from the AOP-KB capture all contents as described in this Users' Handbook sections, the order in which they are presented is different. Specifically, the "snapshots" contain a brief summary of the AOP followed by the overall assessment of the AOP, whereas the detailed description of the KEs and evidence supporting the KERs are presented as appendices (Table 1).

Table 1. Overview of the organisation of content pages in the AOP-KB and reviewer snapshots relative to sections of the Handbook.

\begin{tabular}{|c|c|}
\hline SECTIONS OF AOP-KB AND/OR SNAPSHOT REPORT & $\begin{array}{l}\text { HANDBOOK } \\
\text { SECTION }\end{array}$ \\
\hline AOP Description & Section 1 \\
\hline $\begin{array}{l}\text { Key Event (KE) Descriptions } \\
\text { AOP-KB: Each KE description is on a separate page that } \\
\text { is reached via a link from the KE Table in the AOP } \\
\text { Summary Section } \\
\text { Snapshot: Each KE description is found in Appendix } 1\end{array}$ & Section 2 \\
\hline $\begin{array}{l}\text { Key Event Relationship (KER) Descriptions } \\
\text { AOP-KB: Each KER description is on a separate page }\end{array}$ & Section 3 \\
\hline
\end{tabular}


that is reached via a link from the KER Table in the AOP

Summary Section

Snapshot: Each KER description is found in Appendix 2

Overall Assessment of the AOP

Section 4

See (https://aopwiki.org/info pages/2/info linked pages/5) for the details regarding snapshots created from the $A O P-K B$. 


\section{Introduction to Adverse Outcome Pathways (AOPs)}

An AOP describes a sequence of events commencing with initial interaction(s) of a stressor with a biomolecule within an organism that causes a perturbation in its biology (i.e., molecular initiating event, MIE), which can progress through a dependent series of intermediate key events (KEs) and culminate in an adverse outcome (AO) considered relevant to risk assessment or regulatory decision-making (Table 2). AOPs are typically represented sequentially, moving from one KE to another. In this respect, AOPs define a series of measurable biological changes that can be expected to occur if the perturbation is sufficiently severe (i.e., in terms of potency, duration, frequency) to drive the pathway all the way to the AO. Importantly, AOPs do not describe every detail of the biology but instead focus on describing critical steps or check-points along the path to adversity, which are both measurable and have potential predictive value. While the focus of AOP development is to capture and organise what is known, the process of AOP development may also identify current knowledge gaps which, if filled, could further improve predictive utility.

Table 2. Definitions of key terms and abbreviations used in this Handbook (see AOP guidance for additional terminology relevant to the AOP framework and its application).

\begin{tabular}{|c|c|c|}
\hline $\begin{array}{l}\text { Molecular } \\
\text { initiating } \\
\text { event }\end{array}$ & MIE & $\begin{array}{l}\text { A specialised type of key event that represents the initial } \\
\text { point of chemical/stressor interaction at the molecular level } \\
\text { within the organism that results in a perturbation that starts } \\
\text { the AOP. }\end{array}$ \\
\hline Key event & $\mathrm{KE}$ & $\begin{array}{l}\text { A change in biological or physiological state that is both } \\
\text { measurable and essential to the progression of a defined } \\
\text { biological perturbation leading to a specific adverse } \\
\text { outcome. }\end{array}$ \\
\hline $\begin{array}{l}\text { Key event } \\
\text { relationship }\end{array}$ & KER & $\begin{array}{l}\text { A scientifically-based relationship that connects one key } \\
\text { event to another, defines a causal and predictive relationship } \\
\text { between the upstream and downstream event, and thereby } \\
\text { facilitates inference or extrapolation of the state of the } \\
\text { downstream key event from the known, measured, or } \\
\text { predicted state of the upstream key event. }\end{array}$ \\
\hline $\begin{array}{l}\text { Adverse } \\
\text { Outcome }\end{array}$ & $\mathrm{AO}$ & $\begin{array}{l}\text { A specialised type of key event that is generally accepted as } \\
\text { being of regulatory significance on the basis of } \\
\text { correspondence to an established protection goal or } \\
\text { equivalence to an apical endpoint in an accepted regulatory } \\
\text { guideline toxicity test. }\end{array}$ \\
\hline
\end{tabular}


KEs are measurable biological changes that are essential to the progression along an AOP. Essentiality implies that the KEs play a causal role in the pathway such that if the $\mathrm{KE}$ is prevented or fails to occur, progression to subsequent KEs in the pathway will not occur. While KEs are essential to progression along the AOP, they are not necessarily sufficient. Just because a particular KE is observed, does not mean the perturbation will necessarily progress all the way to the AO. Rather, the conditions under which progression can be expected are described as part of the KERs that link one KE to another in sequence to form an AOP.

The AOP framework provides a transparent and scientifically-based means to organise and present current knowledge of predictable relationships between MIEs, subsequent KEs and AOs. The objective underlying AOP development is to ultimately support inference or extrapolation from one KE to another. Most notably, consistent with the proposed vision for regulatory toxicology in the 21 st century, there is considerable interest in extrapolating from KE measurements that may be made efficiently and costeffectively, typically at low levels of biological organisation, to adverse effects at higher levels of organisation that are relevant to regulatory protection goals and decision-making (Krewski et al. 2010). The overall $\mathrm{WoE}$ and level of certainty underlying the inference and extrapolation will in turn dictate the most suitable application of the AOP.

Assessment of AOPs and evaluation of their suitability for application in different regulatory contexts and the assimilation of the relevant characterisation of supporting biological information relies in part on (1) the confidence and precision with which the KEs can be measured, (2) the level of confidence in the relationships between the KEs linked in an AOP (KERs) based on biological plausibility, and empirical support for the KERs; and (3) WoE for the overall hypothesised pathway, taking into account a number of additional considerations, including any uncertainties and inconsistencies. Therefore, overall assessment of AOPs is best supported by providing thorough descriptions of the KEs [Section 2], relationships between those KEs [i.e., KERs, Section 3], and by robust consideration of supporting evidence for the biological plausibility and empirical support for KERs [Section 3D] and essentiality of KEs [Section 4]. Consequently, the Handbook and AOP-KB are structured in a manner that prompts AOP developers to provide relevant types of supporting information.

\section{Principles of AOP Development and their Implications for AOP Description}

As a pragmatic convention, AOPs are conceptualised as a single sequence of events proceeding from the MIE to the AO via a series of intermediate KEs. That is, they describe how one particular molecular perturbation may cause one AO, not every possible AO that perturbation may cause, nor every way a particular AO may arise. It is recognised that MIEs, KEs, and AOs may be shared by more than one AOP. Consequently, it is desirable to describe KEs as discrete (modular) units without reference to a specific MIE, AO, or other KEs. Likewise, it is useful to describe relationships between discrete pairs of KEs (KERs), without reference to other elements of the AOP. This facilitates generation of generic KE or KER descriptions that can be linked to 
multiple other AOPs. Such an approach both fosters consistency and increases efficiencies in the AOP development process by eliminating the need for AOP developers to completely re-describe biological measurements (KEs) or evidence supporting inference from one KE to another (KERs) that another developer may have already detailed. Maintaining KE and KER descriptions as discrete units that avoid reference to other elements of the AOP also facilitates the updating of KE and KER descriptions as new methods for measuring KEs or new evidence supporting KERs are developed. Finally, it facilitates the construction and conceptualisation of AOP networks.

An AOP network is defined as an assembly of two or more AOPs that share one or more KEs in common. If the components of an AOP (KEs and KERs) are described in a modular fashion, AOP networks emerge from the description of individual AOPs that share KEs. In a network, KEs represent nodes while KERs represent directed edges that link those nodes together. Through their defacto construction as more AOPs are added to the AOP-KB, AOP networks can be viewed as capturing broader knowledge concerning the range of possible AOs a perturbation may cause, or the range of ways in which a particular adverse outcome may occur. AOP networks are also critical for addressing exposures to multiple stressors that lead to the same AO or to individual stressors that perturb multiple MIEs (Knapen et al., 2015; Villeneuve et al., 2014a, b) and for understanding potential interactions between co-occurring AOPs.

In describing the KEs and KERs that make up an AOP, each information field of the KE or KER description should be completed as thoroughly as is practical and supported through citation of primary literature and other references as appropriate. It is recognised that AOP descriptions reflect current knowledge and will evolve as additional information becomes available. In this respect, AOP descriptions should be regarded as "living documents". Not all sections described need to be completed immediately. It is expected that AOPs may have gaps that may be addressed over time as the science progresses or as other researchers contribute. Likewise, collaboration and contributions from other developers is encouraged.

Indeed, AOPs provide a relevant construct to promote collaboration between experts in various areas of research and the regulatory risk assessment community as a basis to better coordinate and tailor research to practical application, such as the development of KE-based testing strategies. Collaboration between a range of experts with expertise in these different areas in the development and assessment of AOPs is therefore strongly encouraged. The AOP-Wiki facilitates this collaboration by providing a tool to organise and share the relevant data and information. Consequently, it is recommended that descriptions are structured in a way that facilitates addition and revision of information as it is developed; for example, through the use of bullets or tables and organisation into topical subsections rather than development of extensive narrative text. Rather than representing a daunting compilation of information that must be assembled to adequately describe an AOP, the sections defined in the Handbook and AOP-KB should be viewed as an organisational structure for assembling a transparent record of scientific support for an AOP and a basis for clear delineation of current gaps in our knowledge. Organisation 
of information in this manner is intended to facilitate collaborative development, transparency, and appropriate use of the information assembled.

In this handbook, particular emphasis is placed on sections of the template related to the description of the MIE, KEs and AO in an AOP (Section 2), the assembly of available scientific evidence supporting the KERs (Section 3) and the summation of the support for the AOP as a whole (Section 4) as a basis to consider its potential application.

If the author aims to publish the AOP in the OECD Series on AOPs, it is strongly encouraged that the text of the AOP description, including the list of references, should conform, to the extent possible, with the OECD Style Guide (https://www.oecd.org/about/publishing/OECD-Style-Guide-Third-Edition.pdf) (OECD, 2015). 


\section{Obtaining Author Access to the AOP-KB}

Read-access to all contents of the AOP-KB (http://aopkb.org/) and the AOP-Wiki (https://aopwiki.org) is publicly available world-wide without need to create a user profile, login ID, or password.

Commentor access: A self-created user account, with a verified email address, grants the user the ability to comment on all pages in the AOP-Wiki including AOPs, KEs, and KERs. Users can create an account on the AOP-Wiki by following the instructions here:

https://aopwiki.org/info_pages/2\#Contributing to the AOP Wiki

Author Access: In order to create or edit AOPs, KEs, or KERs, the user must request author access to the AOP-Wiki by following the instructions here:

https://aopwiki.org/info_pages/2\#Requesting Author Access. 


\section{A Note on AOP Descriptions in the AOP-KB}

AOP descriptions in the AOP-KB can be viewed as consisting of two types of information, structured information and free text.

Structured information is derived from standardised ontologies available through lookup tables or by making selections from a drop-down list. Structured information fields within the AOP-KB populate a back-end database. The terms and information in that database is machine-readable and can be used to aid various computational analyses, querying, and searching of the AOP-KB. For example, construction of AOP networks from the modular units of individual AOP descriptions relies on these structured annotation fields.

Free text sections in the AOP-KB provide AOP developers with much greater descriptive flexibility than structured information fields. While free text is searchable, it is not standardised and machine-readable, and has much more limited use from a computational standpoint.

As a means to balance computational accessibility along with the desire for descriptive accuracy and richness, the AOP-KB incorporates both elements. Consequently, AOP developers are encouraged to complete both the structured information and free text sections of the AOP descriptions to the extent they are able. 


\section{Section 1 - AOP Description}

This section is for information that describes the overall AOP. The information described in section 1 is entered on the upper portion of an AOP page within the AOP-Wiki. This is where some background information may be provided, the structure of the AOP is described, and the KEs and KERs are listed.

\section{AOP Identifier and Title}

This subsection provides guidance for naming the AOP.

\section{AOP Identifier}

Each AOP is automatically given a numerical AOP identifier when it is created.

\section{AOP Title}

Each AOP should be given a descriptive title that takes the form "MIE leading to AO". For example, "Aromatase inhibition [MIE] leading to reproductive dysfunction [AO]" or "Thyroperoxidase inhibition [MIE] leading to decreased cognitive function [AO]". In cases where the MIE is unknown or undefined, the earliest known KE in the chain (i.e., furthest upstream) should be used in lieu of the MIE and it should be made clear that the stated event is a KE and not the MIE.

In some cases, AOPs linking the same MIE to the same AO may proceed through different intermediate KEs. Naming based on MIE and AO alone can result in a series of distinct AOPs with the same title. While these are distinguished in the AOP-KB by their AOP page ID numbers, each of which has a distinct URL, it can be hard for users to discriminate them at a glance. In such cases, an additional descriptor should be added to the title using the form "MIE leading to AO via distinctive KE". For example, "cyclooxygenase inhibition [MIE] leading to reproductive dysfunction [AO] via inhibition of pheromone release" versus "cyclooxygenase inhibition [MIE] leading to reproductive dysfunction $[\mathrm{AO}]$ via interference with spindle assembly checkpoint". 


\section{Short Name}

A short name should also be provided that succinctly summarises the information from the title. This name should not exceed 90 characters.

Implementation in the AOP-Wiki

https://aopwiki.org/info_pages/2/info_linked_pages/1\#A Create a new AOP

\section{Graphical Representation of the AOP}

A graphical summary of the AOP listing all the KEs in sequence, including the MIE (if known) and AO, and the pair-wise relationships (links or KERs) between those KEs should be provided. This is easily achieved using the standard box and arrow AOP diagram (Figure 1).

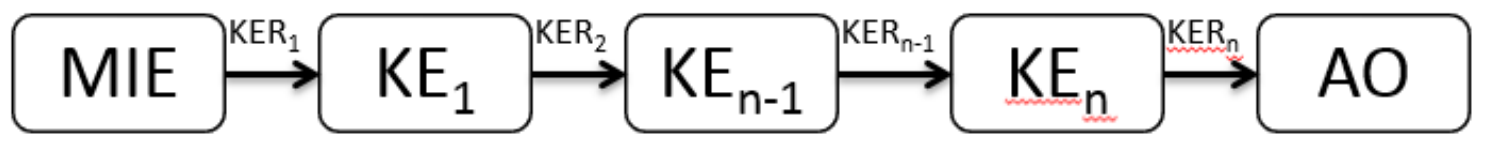

Figure 1. Example of a generic AOP diagram in its simplest form (see also https://aopwiki.org/aops/23\#graphical_representation)

Development tip 1 - Graphical Representation: The graphical representation (AOP diagram) serves as a useful road-map to guide AOP development in the AOP-KB. For this reason, it is recommended that an AOP diagram be developed prior to creating an AOP description in the AOP-KB. Starting with the graphical summary provides a useful overview of the KE and KER pages that will need to be included. Ideally, development of a graphical overview of the AOP to be developed should be followed by a search of existing content to determine whether analogous AOPs and/or synonymous KEs or KERs may already exist in the knowledgebase. This can avoid duplicative effort and help to ensure that KEs and KERs are shared among AOPs, allowing for de facto creation of AOP networks. Once existing KE and KER pages relevant to the AOP have been identified, the developer then knows which pages in the AOP-KB will need to be created de novo.

The graphical summary is prepared and uploaded by the user (templates are available) and is often included as part of the proposal when AOP development projects are submitted to the OECD AOP Development Workplan. 
The graphical representation or AOP diagram provides a useful and concise overview of the KEs that are included in the AOP, and the sequence in which they are linked together. This can aid both the process of development, as well as review and use of the AOP.

Development tip 2 - Number of KEs to include: Determining the number of KEs to include in an AOP and the specificity with which they are defined is one of the more challenging aspects of AOP development. In describing KEs within an AOP, it is important to recognise their distinction from "mechanism of action". AOPs provide a description of a limited number of essential, measurable events (check-points) leading to induction of the relevant toxicity endpoint. They do not necessarily provide a comprehensive molecular description of every aspect of the biology involved. With that in mind, the following "rules of thumb" can help guide the process of KE definition (Villeneuve et al. 2014a, b):

- Where possible and appropriate for application, try to include at least one $\mathrm{KE}$ at each major level of biological organisation (molecular, cellular, tissue, organ, individual).

- Where feasible/appropriate, focus on KEs that can be measured in a relatively routine manner over those that require highly specialised expertise, equipment, or supplies to measure. These will tend to be the KEs for which empirical evidence to support KERs is more likely to be available to support the WoE evaluation.

- Select a limited number of KEs that are measurable and for which evidence supports plausibility and potential predictive utility. Where relevant, more detailed description of the underlying biology involved can be incorporated into the descriptions of the biological plausibility linking two KEs (see section 3 - KER descriptions).

Implementation in the AOP-Wiki:

https://aopwiki.org/info_pages/2/info_linked_pages/1\#B Graphical Representation of the $\underline{\mathrm{AOP}}$

\section{Authors of AOP}

This section provides guidance on author identification.

\section{Authors and Affiliations}

List the name and affiliation information of the individual(s)/organisation(s) that created/developed the AOP. In the context of the OECD AOP Development Workplan, this would typically be the individuals and organisation that submitted an AOP development proposal to the EAGMST. Significant contributors to the AOP should also 
be listed. A corresponding author with contact information may be provided here. This author does not need an account on the AOP-KB and can be distinct from the point of contact below. The list of authors will be included in any snapshot made from an AOP.

\section{Point of Contact}

Indicate the point of contact for the AOP-KB entry itself. This person is responsible for managing the AOP entry in the AOP-KB and controls write access to the page by defining the contributors as described below. Clicking on the name will allow any wiki user to correspond with the point of contact via the email address associated with their user profile in the AOP-KB. This person can be the same as the corresponding author listed in the authors section but isn't required to be. In cases where the individuals are different, the corresponding author would be the appropriate person to contact for scientific issues whereas the point of contact would be the appropriate person to contact about technical issues with the AOP-KB entry itself. Corresponding authors and the point of contact are encouraged to monitor comments on their AOPs and develop or coordinate responses as appropriate.

\section{AOP-KB Contributors}

List user names of all authors contributing to or revising pages in the AOP-KB that are linked to the AOP description. This information is mainly used to control write access to the AOP page and is controlled by the point of contact.

Implementation in the AOP-Wiki:

https://aopwiki.org/info_pages/2/info linked pages/1\#C Authors of AOP

\section{Status and Date Modified}

This section provides guidance on the various status trackers for AOPs.

\section{Author Status}

The status section is used to provide AOP-KB users with information concerning how actively the AOP page is being developed, what type of use or input the authors feel comfortable with given the current level of development, and whether it is part of the OECD AOP Development Workplan and has been reviewed and/or endorsed. "Author Status" is an author defined field that is designated by selecting one of several options from a drop-down menu (Table 3). The "Author Status" field should be changed by the point of contact, as appropriate, as AOP development proceeds. 
Table 3. Drop-down options for "Author status" field

\begin{tabular}{|c|c|}
\hline Selection & Explanation \\
\hline $\begin{array}{l}\text { Under } \\
\text { development: not } \\
\text { open for comment; } \\
\text { Do not cite }\end{array}$ & $\begin{array}{l}\text { This is the default status assigned when a new AOP page is } \\
\text { created in the AOP-Wiki. It is used to indicate that the } \\
\text { project team is actively developing the pages and that the } \\
\text { author(s) have new content they expect to add such that } \\
\text { commenting on or citing the existing content is premature. }\end{array}$ \\
\hline $\begin{array}{l}\text { Open for } \\
\text { comment; do not } \\
\text { cite }\end{array}$ & $\begin{array}{l}\text { This status is used to indicate that the authors have added } \\
\text { the primary content they wish to include and they invite the } \\
\text { community to comment on that content via the Discussion } \\
\text { pages. However, this designation indicates that the authors } \\
\text { do not feel the AOP should be cited in its current form. For } \\
\text { example, perhaps they have identified major uncertainties or } \\
\text { gaps that still need to be addressed. This is a common } \\
\text { designation to use for AOPs that represent a hypothesised } \\
\text { AOP for which supporting evidence has not yet been } \\
\text { assembled. }\end{array}$ \\
\hline $\begin{array}{l}\text { Open for citation } \\
\text { and comment }\end{array}$ & $\begin{array}{l}\text { This status is used to indicate that the author(s) have added } \\
\text { the content they wish to include on their AOP page (and the } \\
\text { associated KE and KER pages) and they invite the } \\
\text { community to comment on that content via the Discussion } \\
\text { pages and cite the AOP in its current form, if desired. This } \\
\text { designation usually indicates that the authors stand behind } \\
\text { their contribution and take responsibility for the scientific } \\
\text { content. }\end{array}$ \\
\hline Open for adoption & $\begin{array}{l}\text { This refers to "adoption" in the sense of new authors taking } \\
\text { over responsibility for further development of the AOP. It } \\
\text { should not be confused with an AOP that should be } \\
\text { considered for endorsement or use. This status is used to } \\
\text { indicate that the primary author(s) of the AOP are no longer } \\
\text { actively working on the page, but would like to invite others } \\
\text { from the community to take-over development of the AOP. } \\
\text { An open for adoption status also signals the curators of the } \\
\text { AOP-Wiki that the authors feel the content provided } \\
\text { warrants further development. AOPs that are open for } \\
\text { adoption will not be deleted from the AOP-KB without first } \\
\text { consulting the current Point of Contact. }\end{array}$ \\
\hline $\begin{array}{l}\text { Not under active } \\
\text { development }\end{array}$ & $\begin{array}{l}\text { This status indicates the primary author(s) of the AOP are } \\
\text { no longer actively working on the page. Others may still } \\
\text { contact the authors about taking-over development of the } \\
\text { pages if desired. However, the content provided may or may } \\
\text { not warrant further development. AOPs with this status }\end{array}$ \\
\hline
\end{tabular}


designation are subject to deletion at the discretion of the curators of the AOP-KB.

\section{OECD Status}

For AOPs that are included in a project that has been accepted into the OECD AOP Development Workplan (see http://www.oecd.org/chemicalsafety/testing/projectsadverse-outcome-pathways.htm), status with regard to progress through OECD review and endorsement processes is tracked by the OECD EAGMST. 'OECD status' tracks the level of review/endorsement the AOP has been subjected to. This designation is managed and updated by the OECD. It is not selected by the AOP author(s).

\section{OECD Project Number}

The OECD project number is also indicated along with the current status of the AOP with regard to the OECD Development Workplan. This designation is managed and updated by the OECD. It is not selected by the AOP author(s).

\section{SAAOP Status}

All AOPs under development in the AOP-KB are monitored by curators who are members of the Society for the Advancement of AOPs (SAAOP). These curators maintain a separate status designation for AOPs based on their evaluation of the current state of the AOP. These designations (Table 4) are managed and updated by the SAAOP curators. They are not selected by the AOP author(s). Currently the SAAOP status list includes the following:

Table 4. Explanation for SAAOP status

\begin{tabular}{|c|c|}
\hline SAAOP Status & Explanation \\
\hline Included in the & An AOP development project proposal has been reviewed by \\
\hline $\begin{array}{l}\text { OECD work } \\
\text { plan }\end{array}$ & $\begin{array}{l}\text { OECD EAGMST, accepted into the workplan, and a project } \\
\text { number assigned. }\end{array}$ \\
\hline Proposed for & A SAAOP curator has encouraged the author to submit a \\
\hline $\begin{array}{l}\text { OECD work } \\
\text { plan }\end{array}$ & $\begin{array}{l}\text { proposal to OECD. Indicates well developed content that is } \\
\text { likely suitable for review. }\end{array}$ \\
\hline $\begin{array}{l}\text { Under } \\
\text { development }\end{array}$ & $\begin{array}{l}\text { Indicates the SAAOP views the content as still under } \\
\text { development and not ready for formal review. }\end{array}$ \\
\hline Archive & $\begin{array}{l}\text { Indicates that the entry is likely to be deleted. AOPs with an } \\
\text { archived status are not listed when a user is browsing the AOPs } \\
\text { but they will show up when a search is made. This is typically } \\
\text { for AOPs that are not under active development and not suitable } \\
\text { for adoption. }\end{array}$ \\
\hline
\end{tabular}

Implementation in the AOP-Wiki:

https://aopwiki.org/info_pages/2/info_linked_pages/1\#D Status of an AOP 


\section{Date Modified}

The date the AOP was last modified is automatically tracked by the AOP-KB. The date modified field can be used to evaluate how actively the page is under development and how recently the version within the AOP-Wiki has been updated compared to any snapshots that were generated.

Implementation in the AOP-Wiki:

https://aopwiki.org/info_pages/2/info_linked_pages/1\#History of Modifications

\section{Abstract}

In the abstract section, authors should provide a concise and informative summation of the AOP under development that can stand-alone from the AOP page. Abstracts should typically be 200-400 words in length (similar to an abstract for a journal article). Suggested content for the abstract includes the following: (1) the background/purpose for initiation of the AOP's development (if there was a specific intent); (2) a brief description of the MIE, AO, and/or major KEs that define the pathway; (3) a short summation of the overall WoE supporting the AOP and identification of major knowledge gaps (if any); (4) if a brief statement about how the AOP may be applied (optional). The aim is to capture the highlights of the AOP and its potential scientific and regulatory relevance.

Implementation in the AOP-Wiki:

https://aopwiki.org/info_pages/2/info_linked_pages/1\#E AOP Abstract

https://aopwiki.org/info_pages/2/info_linked_pages/1 05\%20To\%20edit\%20AOP\%20abstract

\section{Background (Optional)}

This optional subsection should be used to provide background information for AOP reviewers and users that is considered helpful in understanding the biology underlying the AOP and the motivation for its development. The background should NOT provide an overview of the AOP, its KEs or KERs, which are captured in more detail below. A few examples of potential uses of the optional background section include:

If the AOP was a result of research funded through a particular grant or research program, the authors may want to provide information regarding the source of funding for the research that led to development of the AOP and the scope and key research questions the over-arching research effort was designed to address. 
If the AOP is one of a series of related AOPs that the author(s) developed as part of a network-guided approach to AOP development, the authors may want to make explicit reference to other AOPs that were also developed as part of the effort in this section.

In the case of AOPs that were developed as a regulatory application case study or to support a particular regulatory decision, the authors may want to provide a bit of background on the problem formulation that motivated development of the AOP.

If there is some particularly interesting biology that is encompassed by the AOP that is not necessarily evident from the KE and KER descriptions, but would likely be of interest to other investigators with an interest in the AOP, those details could be provided here.

In general, this section is suitable for any additional information that does not necessarily fit in other parts of the AOP description, but may be of interest to readers/users.

Implementation in the AOP-Wiki:

https://aopwiki.org/info_pages/2/info_linked_pages/1\#F Background Information

\section{KE and KER Tables}

Tables listing each KE and KER are automatically created in the AOP-KB as KE pages to link to the AOP are selected or created and as KERs are defined.

a. KE Table: This table summarises all of the KEs of the AOP, including the MIE and AO. This table is populated in the AOP-Wiki as KEs are added to the AOP. Each table entry acts as a link to the individual KE description page. For guidance on completing the KE descriptions see Section 2.

b. KER Table: This table summarises all of the KERs of the AOP and is populated in the AOP-Wiki as KERs are added to the AOP. Each table entry acts as a link to the individual KER description page. For guidance on completing the KER descriptions see Section 3.

Implementation in the AOP-Wiki:

https://aopwiki.org/info_pages/2/info_linked_pages/1\#G KE and KER Tables

\section{Network View}

The AOP-Wiki automatically generates a network view of the AOP. This network graphic is based on the information provided in the MIE, KEs, AO, KERs and WoE summary tables. The width of the edges representing the KERs is determined by its WoE 
confidence level, with thicker lines representing higher degrees of confidence. This network view also shows which KEs are shared with other AOPs.

Examples in the AOP Wiki:

https://aopwiki.org/info_pages/2/info_linked_pages/1\#H AOP Networks

https://aopwiki.org/aops/15\#network view

https://aopwiki.org/aops/23\#network_view

https://aopwiki.org/aops/38\#network view

\section{Stressors}

The stressor field is a structured data field that can be used to annotate an AOP with standardised terms identifying stressors known to trigger the MIE/AOP. Most often these are chemical names selected from established chemical ontologies. However, depending on the information available, this could also refer to chemical categories (i.e., groups of chemicals with defined structural features known to trigger the MIE). It can also include non-chemical stressors such as genetic or environmental factors. Although AOPs themselves are not chemical or stressor-specific, linking to stressor terms known to be relevant to different AOPs can aid users in searching for AOPs that may be relevant to a given stressor.

Implementation in the AOP-Wiki:

https://aopwiki.org/info_pages/2/info_linked_pages/1\#Stressors 


\section{Section 2 - KE Descriptions}

\section{Development tip 3 - Sharing of KEs:}

- Use existing KEs when possible - when adding KEs to an AOP it is strongly recommended to use KEs that already exist in the AOP-KB as much as possible.

- Existing KE requires modification - If an existing KE requires modification to make it suitable, changes to the content on that page should be coordinated with the point(s) of contact for other AOPs sharing the KE to ensure that the original meaning is not altered.

- Creating new KEs - If no suitable KEs are available in the knowledgebase, or if the revisions needed to make an existing KE description suitable for the AOP under-development would make it unsuitable for use in AOPs it is already linked to, then a new KE should be created.

- AOP-KB Etiquette - When using an existing KE, it is the responsibility of the person making changes to ensure that KEs used in multiple AOPs are not altered in such a way as to diminish the applicability of that KE for the existing AOPs. Please be courteous to your fellow AOP developers.

Implementation in the AOP-Wiki:

https://aopwiki.org/info pages/2/info linked_pages/3\#A-D Create a New Key Event

KE ID

When a KE is created, an ID number is automatically assigned to it. This number is used for tracking the KE in the AOP-KB.

\section{KE Title}

The KE title should describe a discrete biological change that can be measured. It should generally define the biological object or process being measured and whether it is increased, decreased, or otherwise definably altered relative to a control state. For example "enzyme activity, decreased", "hormone concentration, increased", or "growth rate, decreased", where the specific enzyme or hormone being measured is defined. 


\section{Short Name}

The KE short name should be a reasonable abbreviation of the KE title and is used in labelling this object throughout the AOP-Wiki. The short name should be less than 80 characters in length.

\section{Level of Biological Organisation}

Structured terms, selected from a drop-down menu, are used to identify the level of biological organisation for each KE. Note, KEs should be defined within a particular level of biological organisation. Only KERs should be used to transition from one level of organisation to another. Selection of the level of biological organisation defines which structured terms will be available to select when defining the Event Components (below).

\section{KE Components and Biological Context}

Because one of the aims of the AOP-KB is to facilitate de facto construction of AOP networks through the use of shared KE and KER elements, authors are also asked to define their KEs using a set of structured ontology terms (Event Components). In the absence of structured terms, the same KE can readily be defined using a number of synonymous titles (read by a computer as character strings). In order to make these synonymous KEs more machine-readable, KEs should also be defined by one or more "event components" consisting of a biological process, object, and action with each term originating from one of 22 biological ontologies (Ives, et al., 2017; https://aopwiki.org/info_pages/2/info_linked_pages/7\#List). Biological process describes dynamics of the underlying biological system (e.g., receptor signalling). The biological object is the subject of the perturbation (e.g., a specific biological receptor that is activated or inhibited). Action represents the direction of perturbation of this system (generally increased or decreased; e.g., 'decreased' in the case of a receptor that is inhibited to indicate a decrease in the signalling by that receptor).

\section{Development tip 4 - How specifically should my KEs be defined:}

The following are some general recommendations and "rules of thumb" concerning how specifically to define a KE (see also Villeneuve et al. 2014a, b):

- Define the KE with enough specificity that one would know what to measure to determine the state of the KE. For example "histological changes" is too broad; "oocyte atresia" or "hyperplasia" would be better.

- KEs should refer to/focus on a single measurable event within a specific biological level of organisation, rather than compounding events together. For example, it would be better to define a KE as "enzyme activity, increased" (if that can be measured), rather than "transcription and translation leading to enzyme activity, increased".

- The biological context of the KE (e.g., the tissue type/taxa/life stage/sex etc.) 
should only be restricted (e.g., "enzyme activity in liver, decreased" or "hormone concentration in females, increased") to the extent that function changes with context. If the function is equivalent in both sexes, do not restrict the context by sex. If the function is equivalent in all cell types, do not restrict to a specific cell type.

Implementation in the AOP-Wiki:

https://aopwiki.org/info_pages/2/info_linked_pages/3\#E Event Components and Biological Context

\section{Other AOPs that use this KE}

All of the AOPs that are linked to this KE will automatically be listed in this subsection. This table can be particularly useful for derivation of AOP networks including the KE.

Implementation in the AOP-Wiki:

https://aopwiki.org/info_pages/2/info linked pages/3\#F AOP

Informationhttps://aopwiki.org/info_pages/2/info_linked_pages/3 - E Event Components and Biological Context

\section{KE Description}

A description of the biological state being observed or measured, the biological compartment in which it is measured, and its general role in the biology should be provided. For example, the biological state being measured could be the activity of an enzyme, the expression of a gene or abundance of an mRNA transcript, the concentration of a hormone or protein, neuronal activity, heart rate, etc. The biological compartment may be a particular cell type, tissue, organ, fluid (e.g., plasma, cerebrospinal fluid), etc. The role in the biology could describe the reaction that an enzyme catalyses and the role of that reaction within a given metabolic pathway; the protein that a gene or mRNA transcript codes for and the function of that protein; the function of a hormone in a given target tissue, physiological function of an organ, etc. Careful attention should be taken to avoid reference to other KEs, KERs or AOPs. Only describe this KE as a single isolated measurable event/state. This will ensure that the KE is modular and can be used by other AOPs, thereby facilitating construction of AOP networks.

Implementation in the AOP-Wiki:

https://aopwiki.org/info_pages/2/info_linked_pages/3\#Key Event Description 


\section{How it is Measured or Detected}

One of the primary considerations in evaluating AOPs is the relevance and reliability of the methods with which the KEs can be measured. The aim of this section of the KE description is not to provide detailed protocols, but rather to capture, in a sentence or two, per method, the type(s) of measurements that can be employed to evaluate the KE and the relative level of scientific confidence in those measurements. Methods that can be used to detect or measure the biological state represented in the KE should be briefly described and/or cited. These can range from citation of specific validated test guidelines, citation of specific methods published in the peer reviewed literature, or outlines of a general protocol or approach (e.g., a protein may be measured by ELISA).

Key considerations regarding scientific confidence in the measurement approach include whether the assay is fit for purpose, whether it provides a direct or indirect measure of the biological state in question, whether it is repeatable and reproducible, and the extent to which it is accepted in the scientific and/or regulatory community. Information can be obtained from the OECD Test Guidelines website (http://www.oecd.org/chemicalsafety/testing/oecdguidelinesforthetestingofchemicals.htm ) and the EURL ECVAM Database Service on Alternative Methods to Animal Experimentation (DB-ALM) (https://ecvam-dbalm.jrc.ec.europa.eu/).

Implementation in the AOP-Wiki:

https://aopwiki.org/info_pages/2/info_linked_pages/3\#How it is Measured or Detected

\section{Biological Domain of Applicability}

The relevant biological domain(s) of applicability of the KE in terms of sex, life-stage, taxa, and other aspects of biological context are defined in this section. In essence, the taxa/life-stage/sex applicability is defined based on the groups of organisms for which the measurements represented by the KEs can feasibly be made with the same functional interpretation.

Defining the taxonomic, life stage and sex relevance of each KE helps to bound the domain of applicability of the AOP as a whole and provides an understanding of how broadly data represented by a KE measurement may be extrapolated, including potential human relevance. As a general guide, there are two primary considerations associated with defining the applicability domain of a KE:

1. Structure: Is the biological object being measured/observed present/conserved in the taxa/sex/life-stage of interest? Here biological object may refer to a protein, a cell type, an organ, etc. 
2. Function: Is the function of that biological object and the process being measured via the KE conserved and relevant in the taxa/sex/life-stage of interest. Does it play the same role?

For example, if the KE involves binding to the estrogen receptor, but invertebrates lack a functional homolog of the estrogen receptor, one could reasonably conclude that the AOP is not relevant to invertebrates on the basis of a lack of conserved structure. Likewise, if the KE involves a measurement in ovary tissue, its applicability domain in terms of sex would be restricted to females. If a $\mathrm{KE}$ involves altered organogenesis (e.g., heart formation), the KE would only be relevant to the life-stage during which the heart is actually formed, and not to the adult life-stage in which organ development has already completed.

Biological Domain of applicability is defined in the AOP-KB using a combination of structured fields and free text. Structured terms can be selected to identify the taxa, life stage, sex, and the level of biological organisation (e.g., cell, tissue or organ) for which the KE is known to be applicable. Selection of structured terms to describe the applicability domain can aid AOP network construction as well as facilitating other types of computational processing and searching of information captured in the AOP-KB.

At the time that developers select structured ontology terms to help define the domain of applicability of the KE, there is also an option to make evidence calls related to applicability of the specific KE in question to that category term. These calls should be based on expert knowledge of the biology and the extent of supporting experimental evidence. Recommendations for these calls are:

- Low: With the understanding that by definition a KE must be measurable in the species/taxonomic group/lifestage/sex defined, no such measurements have been reported or shown experimentally to date;

- $\quad$ Moderate: The measurement associated with the KE can plausibly be made for the species/taxonomic group/lifestage/sex, and there is at least some supporting experimental evidence, although that may be something other than direct measurement of the KE;

- High: The measurement associated with the KE has been made repeatedly or frequently and/or with multiple orthogonal methods for the species/taxonomic group/lifestage/sex.

\section{Taxonomic Applicability}

Latin or common names of a species or broader taxonomic grouping (e.g., class, order, family) can be selected from an ontology. In many cases, individual species identified in these structured fields will be those for which the strongest evidence used in constructing the AOP was available in relation to this KE. 


\section{Life Stage Applicability}

The structured ontology terms for life-stage are more comprehensive than those for taxa, but may still require further description/development and explanation in the free text section.

\section{Sex Applicability}

The authors must select from one of the following: Male, female, mixed, asexual, third gender, hermaphrodite, or unspecific.

\section{Evidence for Biological Domain of Applicability}

This free text section should be used to elaborate on the scientific basis for the indicated domains of applicability and the WoE calls (if provided). While structured terms may be selected to define the taxonomic, life stage and sex applicability (see structured applicability terms, above) of the KE, the structured terms may not adequately reflect or capture the overall biological applicability domain (particularly with regard to taxa). Likewise, the structured terms do not provide an explanation or rationale for the selection. The free-text section on evidence for taxonomic, life stage, and sex applicability can be used to elaborate on why the specific structured terms were selected, and provide supporting references and background information.

Implementation in the AOP-Wiki:

https://aopwiki.org/info_pages/2/info_linked_pages/3\#I Biological Domain of Applicability for KE

\section{MIE-Specific Content}

An MIE is a specialised KE that represents the beginning (point of interaction between a stressor and the biological system) of an AOP. Description of an MIE should include all the information listed above for KEs and also requires two additional fields of information: evidence that the event can be triggered by a chemical (or other stressor), and a list of known stressors. If the KE is being described is not an MIE, simply indicate "not an MIE" in this section

Implementation in the AOP-Wiki:

https://aopwiki.org/info_pages/2/info_linked_pages/3\#J MIE-Specific Content 


\section{Evidence for Perturbation of MIE by Stressor}

The MIE involves a chemical interaction (e.g., a reaction, covalent binding, hydrogen bonding, electrostatic interaction, etc.) between a chemical stressor and chemically defined biomolecules within an organism. In some cases, this may be a highly specific interaction, for example between an exogenous ligand and a specific receptor. In other cases, it may be non-specific, as in the case of a reactive chemical that can covalently modify a wide array of proteins. In still other cases, non-chemical stressors of various types may initiate a biological perturbation through interaction with a defined biological target (for example actions of a virus in a host cell, physical damage to gill tissue in a fish, effects of UV radiation on DNA, etc.). Any of these cases can be described as an MIE, provided that the general nature of the stressor-biomolecule interaction is understood. Therefore, when a specific MIE can be defined (i.e., the molecular target and nature of interaction is known), in addition to describing the biological state associated with the MIE, how it can be measured, and its taxonomic, life stage, and sex applicability, it is useful to list stressors known to trigger the MIE and provide evidence supporting that initiation. This will often be a list of prototypical compounds demonstrated to interact with the target molecule in the manner detailed in the MIE description to initiate a given pathway (e.g., 2,3,7,8-TCDD as a prototypical AhR agonist; 17 $\alpha$-ethynyl estradiol as a prototypical ER agonist). Depending on the information available, this could also refer to chemical categories (i.e., groups of chemicals with defined structural features known to trigger the MIE). Known stressors should be included in the MIE description, but it is not expected to include a comprehensive list. Rather initially, stressors identified will be exemplary and the stressor list will be expanded over time.

\section{Stressors}

This is a structured field used to identify specific agents (generally chemicals) that can trigger the KE. Stressors identified in this field will be linked to the KE in a machinereadable manner, such that, for example, a stressor search would identify this as an event the stressor can trigger. NOTE: intermediate or downstream KEs in one AOP may function as MIEs in other AOPs, meaning that stressor information may be added to the $\mathrm{KE}$ description, even if it is a downstream $\mathrm{KE}$ in the pathway currently under development.

Information concerning the stressors that may trigger an MIE can be defined using a combination of structured and unstructured (free-text) fields. For example, structured fields may be used to indicate specific chemicals for which there is evidence of an interaction relevant to this MIE. By linking the KE description to a structured chemical name, it will be increasingly possible to link the MIE to other sources of chemical data and information, enhancing searchability and inter-operability among different datasources and knowledgebases. The free-text section "Evidence for perturbation of this MIE by stressor" can be used both to identify the supporting evidence for specific stressors triggering the MIE as well as to define broad chemical categories or other properties that classify the stressors able to trigger the MIE for which specific structured terms may not exist. 


\section{AO-Specific Content}

An $\mathrm{AO}$ is a specialised $\mathrm{KE}$ that represents the end (an adverse outcome of regulatory significance) of an AOP. For KEs that are designated as an AO, one additional field of information (regulatory significance of the $\mathrm{AO}$ ) should be completed, to the extent feasible. If the KE is being described is not an AO, simply indicate "not an AO" in this section.

Implementation in the AOP-Wiki:

https://aopwiki.org/info_pages/2/info_linked_pages/3\#K AO-Specific Content

\section{Regulatory Significance of the AO}

A key criterion for defining an $\mathrm{AO}$ is its relevance for regulatory decision-making (i.e., it corresponds to an accepted protection goal or common apical endpoint in an established regulatory guideline study). For example, in humans this may constitute increased risk of disease-related pathology in a particular organ or organ system in an individual or in either the entire or a specified subset of the population. In wildlife, this will most often be an outcome of demographic significance that has meaning in terms of estimates of population sustainability. Given this consideration, in addition to describing the biological state associated with the AO, how it can be measured, and its taxonomic, life stage, and sex applicability, it is useful to describe regulatory examples using this AO.

\section{References}

List of the literature that was cited for this KE description. Ideally, the list of references, should conform, to the extent possible, with the OECD Style Guide (https://www.oecd.org/about/publishing/OECD-Style-Guide-Third-Edition.pdf) (OECD, 2015). 


\section{Section 3 - KER Descriptions}

The utility of AOPs for regulatory application is defined, to a large extent, by the confidence and precision with which they facilitate extrapolation of data measured at low levels of biological organisation to predicted outcomes at higher levels of organisation and the extent to which they can link biological effect measurements to their specific causes. Within the AOP framework, the predictive relationships that facilitate extrapolation are represented by the KERs. Consequently, the overall WoE for an AOP is a reflection in part, of the level of confidence in the underlying series of KERs it encompasses. Therefore, describing the KERs in an AOP involves assembling and organising the types of information and evidence that defines the scientific basis for inferring the probable change in, or state of, a downstream KE from the known or measured state of an upstream KE. Before describing a KER, carefully consider the following guidance:

KERs are always described in the form of a directed relationship (one-way arrow) linking an upstream "causing" event to a downstream "responding" event. The pair of KEs linked via a KER may either be adjacent to one another in the sequence of KEs that define a given AOP, or non-adjacent. Regardless of adjacency, one event is always positioned upstream of the other. By convention (and for clarity), KERs linking adjacent KEs in an AOP are represented using solid arrows, while KERs that link KEs that are not adjacent to one another in sequence are linked via dashed arrows (e.g., Figure 2). This is a graphical convention only which has no bearing on the type of content to include in the KER description.

A KER description has to be created for each adjacent upstream-downstream pair of KEs in the pathway. Graphically speaking, there should always be at least one solid arrow path connecting each KE in the pathway into a sequence. There should be no KEs that are unconnected or are only connected via a non-adjacent path (represented as a dashed arrow) only.

Inclusion and description of non-adjacent KERs within an AOP can be particularly useful for assembling evidence supporting the AOP. For example, some KE measurements may be fairly difficult to make, such that they are rarely made in routine studies. While there may be sufficient data or plausibility to establish an intermediate KE as part of the AOP, much of the available WoE may ignore or "leap over" that particular KE. Including KER descriptions for non-adjacent KE pairs allows the WoE for these relationships to be readily described and linked to other AOPs without compromising the principle of modularity with regard to the KER descriptions. With this in mind, the upstreamdownstream pair of KEs linked via a KER may be adjacent in one AOP and non-adjacent 
in another (Figure 3A). In cases where the upstream-downstream sequence was reversed, a separate KER would be described for each upstream-downstream orientation (Figure 3B).

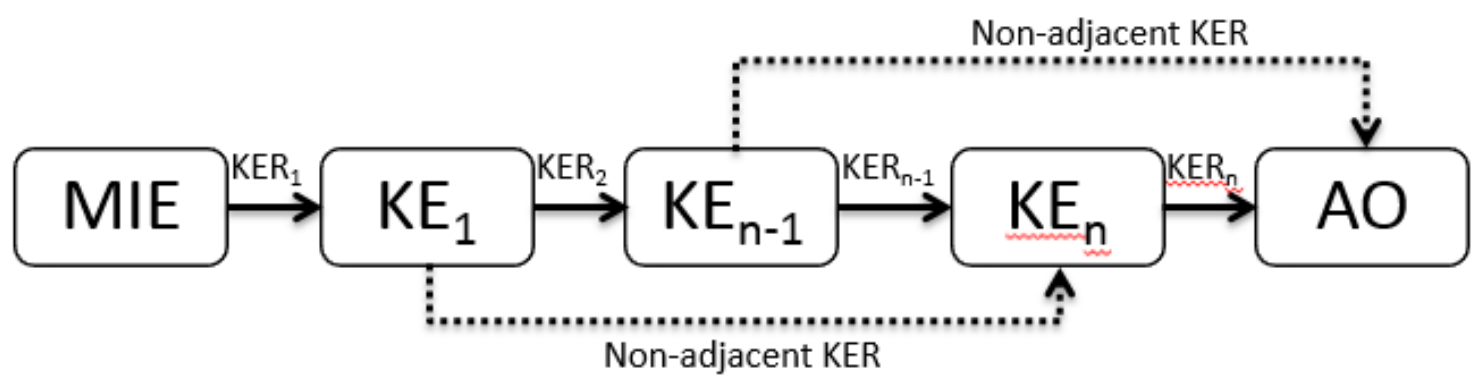

Figure 2. Generic AOP diagram illustrating the graphical convention for depicting KERs linking adjacent (solid arrow) versus non-adjacent (dashed arrow) upstream-downstream KE pairs within an AOP. Regardless of adjacency, each KER represents a predictive relationship between a pair of KEs and can be supported by WoE. Each AOP diagram should portray at least one direct path sequence through all KEs in the pathway (a solid arrow path from MIE to AO, connecting all KEs in the pathway). It is not necessary to create a KER for every non-adjacent pairing, although that can be done if the available supporting evidence warrants.

(A)

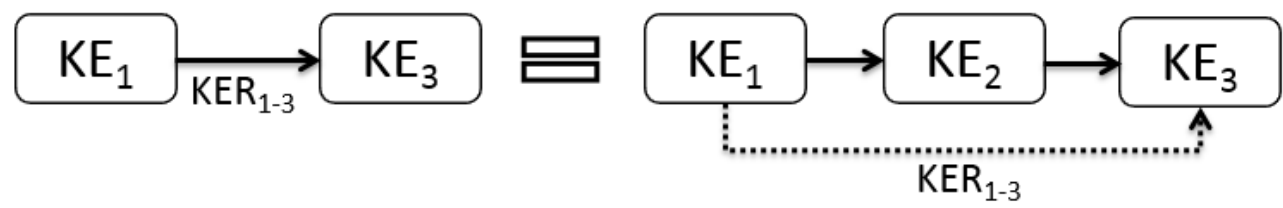

(B)
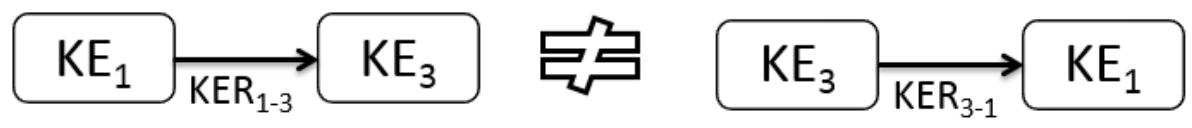

Figure 3. Graphical depiction of the modular functionality of KERs connecting KE1 to KE3. In case (A), the content of KER1-3 is identical despite the fact that the KE1 and KE3 are adjacent in one AOP and non-adjacent in the other. In case (B), KER1-3 is not equivalent to KER3-1. They would be represented as separate pages in the AOP-KB supported by different KER descriptions and evidence.

Overall, the subsections of the KER descriptions are intended to aid the user in collecting relevant information that will support evaluation of the level of confidence in each KER, which in turn contributes to the assessment of the WoE of the AOP overall (section 4). 
Implementation in the AOP-Wiki:

https://aopwiki.org/info pages/2/info linked pages/2\#A-C Create a New Key Event Relationship

\section{KER ID}

When a KER is created, an ID number is automatically assigned to it. This number is used for tracking the KER in the AOP-KB.

\section{KER Title}

The title of the KER should clearly define the two KEs being considered and the sequential relationship between them (i.e., which is upstream and which is downstream). Consequently all KER titles take the form "upstream KE leads to downstream KE".

\section{AOPs Referencing Relationship}

All of the AOPs that are linked to this KER will automatically be listed in this subsection.

\section{Biological Domain of Applicability}

Developers have the option to select one or more structured terms that help to define the biological applicability domain of the KER. In general, this will be dictated by the more restrictive of the two KEs being linked together by the KER. For example, if the upstream $\mathrm{KE}$ is relevant to all vertebrates but the downstream $\mathrm{KE}$ is relevant only to sexually mature, egg-laying female vertebrates, the KER would be relevant to sexually mature egg-laying female vertebrates. Generally speaking, the biological domain of applicability of a KER can never be broader than the more restrictive of the two KEs it links together. Thus, the biological applicability domains of the two KEs being linked is a strong determinant of the biological domain of applicability of a KER. However, in some cases, the biological applicability domain of the KER may be even more restrictive. This is because in addition to structural and functional conservation, the KER also considers the conservation of a regulatory relationship between two KEs. That is, KEupstream has to regulate KEdownstream. Therefore, with regard to KERs, the three considerations that generally guide definition of the biological domain of applicability are:

1. Structure: Are the biological object(s) being measured/observed in the context of the two KEs being linked present/conserved in the taxa/sex/life-stage of interest?

2. Function: Are the functions of those biological objects and the processes being measured in the two KEs conserved and relevant in the taxa/sex/life-stage of interest? Does the object/process play the same role? 
3. Regulation: Is the regulation of the KEdownstream by KEupstream conserved and relevant in the taxa/sex/life-stage of interest?

Selection of structured terms to describe the biological applicability domain can aid AOP network construction as well as facilitating other types of computational processing and searching of information captured in the AOP-Wiki.

Upon selection of structured biological applicability domain terms, developers have the option to classify the extent of the supporting evidence for the terms they have selected:

- Low the relationship is biologically plausible, but hasn't been shown experimentally in this species/taxonomic group/lifestage/sex;

- Moderate the relationship is biologically plausible, and there is some limited supporting experimental evidence in the species/taxonomic group/lifestage/sex of interest;

- High the relationship is biologically plausible, and there is considerable supporting evidence in the species/taxonomic group/lifestage/sex, including evidence of temporal, dose-response, and/or incidence concordance between the two KEs for the group in question.

Implementation in the AOP-Wiki:

https://aopwiki.org/info pages/2/info linked pages/2\#D Biological Domain of

Applicability for KER

\section{Taxonomic Applicability}

Authors can indicate the relevant taxa for this KER in this subsection. The process is similar to what is described for KEs (Section 2).

\section{Life Stage Applicability}

Authors can indicate the relevant life stage for this KER in this subsection. The process is similar to what is described for KEs (Section 2).

\section{Sex Applicability}

Authors can indicate the relevant sex for this KER in this subsection. The process is similar to what is described for KEs (Section 2).

\section{Evidence Supporting the Biological Domain of Applicability}

As for the KEs, there is also a free-text section of the KER description that the developer can use to explain his/her rationale for the structured terms selected with regard to 
taxonomic, life stage, or sex applicability, or provide a more generalizable or nuanced description of the applicability domain than may be feasible using standardised terms.

\section{KER Description}

Provide a brief, descriptive summation of the KER. While the title itself is fairly descriptive, this section can provide details that aren't inherent in the description of the KEs themselves (see Section 5, recommendations regarding number of KEs to include). For example, if the upstream KE was antagonism of a specific receptor, the description could stipulate that "persistent antagonism of the receptor for a period of days" will trigger the downstream KE. Shorter term antagonism of the same receptor (i.e., same upstream KE) may trigger a different downstream KE, and thus would be described as a different KER. This description section can be viewed as providing the increased specificity in the nature of upstream perturbation (KEupstream) that leads to a particular downstream perturbation (KEdownstream), while allowing the KE descriptions to remain generalised so they can be linked to different AOPs. The description is also intended to provide a concise overview for readers who may want a brief summation, without needing to read through the detailed support for the relationship (covered below). Careful attention should be taken to avoid reference to other KEs that are not part of this KER, other KERs or other AOPs. This will ensure that the KER is modular and can be used by other AOPs

Implementation in the AOP-Wiki:

https://aopwiki.org/info_pages/2/info_linked_pages/2\#E Describe the KER

\section{Evidence Supporting this KER}

Assembly and description of the scientific evidence supporting KERs in an AOP is an important step in the AOP development process that sets the stage for overall assessment of the AOP (Section 4). To do this, biological plausibility, empirical support, and the current quantitative understanding of the KER are evaluated with regard to the predictive relationships/associations between defined pairs of KEs as a basis for considering WoE (Section 4). In addition, uncertainties and inconsistencies are considered.

Implementation in the AOP-Wiki:

https://aopwiki.org/info_pages/2/info_linked_pages/2\#F Evidence Supporting this KER

\section{Biological Plausibility}

Define, in free text, the biological rationale for a connection between KEupstream and KEdownstream. What are the structural or functional relationships between the KEs? For example, there is a functional relationship between an enzyme's activity and the product of a reaction it catalyses. 
Supporting references should be included. However, it is recognised that there may be cases where the biological relationship between two KEs is very well established, to the extent that it is widely accepted and consistently supported by so much literature that it is unnecessary and impractical to cite the relevant primary literature. Citation of review articles or other secondary sources, like text books, may be reasonable in such cases. The primary intent is to provide scientifically credible support for the structural and/or functional relationship between the pair of KEs if one is known.

In general, the structural and/or functional relationship supporting plausibility is based on understanding of normal biological function, rather than response to a specific stressor. The description of biological plausibility can also incorporate additional mechanistic detail that helps inform the relationship between KEs, but is not practical/pragmatic to represent as separate KEs due to the difficulty or relative infrequency with which it is likely to be measured. For example, in the case of $\mathrm{G}$ protein coupled receptor activation (KEupstream) leading to increased activity of a specific enzyme (KEdownstream), there may be numerous mechanistic steps in between those KEs (e.g., alterations in signal transduction pathways, transcriptional regulation, post-translational modifications, etc.). These underlying details, if known, can be captured in the description of biological plausibility (if desired) rather than represented as independent KEs. The KER descriptions are an appropriate place for "compounding" or "embedding" that type of biological detail without compromising the reusability of KE descriptions within the AOP-Wiki. However, it should be kept in mind that added detail should only be included to the extent that it enhances the predictive utility of the AOP. In part, the AOP is intended to filter through much of the "biological noise" to focus on what is causally related to the adversity. Thus, efforts should be made to keep the descriptions focused.

\section{Empirical Evidence}

In this section authors are encouraged to cite specific evidence that supports the idea that a change in the upstream KE (KEupstream) will lead to, or is associated with, a subsequent change in the downstream KE (KEdownstream), assuming the perturbation of KEupstream is sufficient.

In particular, it is useful to cite evidence showing that stressors that perturb KEupstream also perturb KEdownstream. Because this section of the KER description cites evidence from specific studies, it is also helpful to provide as much detail about the toxicological and biological context in which the measurements were made, as is feasible, including the stressor(s) tested, the effective doses at each KE, etc. While the KER itself is not intended to be stressor-specific, those details can aid the overall assessment of the individual AOPs that include that KER. These details also help inform the question of consistency of supporting data, consistency across different biological contexts for which the KER is relevant, and the applicability domain of the KER. However, authors are cautioned that this evidence should focus on data that only relate KEupstream to KEdownstream, and should avoid reference to other KEs, KERs and AOPs as much as possible in order to maintain modularity of the KER. 
Given the likelihood that new empirical support will be developed over time, particularly as various AOPs are tested and applied, it is most practical to provide empirical support in the form of bulleted lists or tables that include a short description of the nature of the empirical support along with the corresponding reference(s).

\section{Dose Concordance}

In the case of dose-response concordance, the aim is not to show dose-dependence of a single $\mathrm{KE}$ in the pair, but rather to establish that KEupstream is generally impacted at doses (or stressor severities) equal to or lower than those at which KEdownstream is impacted.

\section{Temporal Concordance}

In the case of temporal concordance, it is desirable to assemble evidence showing that effects on KE upstream are observed earlier in a time-course than effects on the downstream KE.

\section{Incidence Concordance}

In the case of incidence concordance, evidence should be assembled that addresses whether, at an equivalent dose or stressor severity, KEupstream occurs more frequently than KEdownstream .

\section{Other Evidence (optional)}

Although evidence that demonstrates dose, temporal or incidence concordance are preferred, other evidence that empirically supports the relations that a sufficient change in KEupstream will lead to a change in KEdownstream, but do not fall into the above three categories, can be cited in this subsection.

\section{Uncertainties and Inconsistencies}

In addition to outlining the evidence supporting a particular linkage, it is also important to identify inconsistencies or uncertainties in the relationship. This could include, for example, empirical evidence showing changes in KEupstream that did not elicit alterations in KEdownstream. It could also include descriptions of gaps in biological understanding that lend to uncertainties in understanding of the exact nature of the structural or functional relationship between the two KEs. Additionally, while there are expected patterns of concordance that support a causal linkage between the KEs in the pair, it is also helpful to identify experimental details that may explain apparent deviations from the expected patterns of concordance. An example of this would be a case where methods for measuring the upstream KE are relatively insensitive compared 
to those for measuring the downstream KE, leading to the appearance of dose-response or incidence discordance that is simply an artefact of the measurement techniques employed. In this regard, when assembling information from multiple disparate studies, it is important to capture variables that directly influence how well concordance can be assessed (i.e., information regarding the doses tested in various experiments and the timepoints at which various KE measurements were made). Identification of uncertainties and inconsistencies contributes to evaluation of the overall WoE supporting the AOPs that contain a given KER (see Section 4) and to the identification of research gaps that warrant investigation.

Given that AOPs are intended to support regulatory applications, AOP developers should focus on those inconsistencies or gaps that would have a direct bearing or impact on the confidence in the KER and its use as a basis for inference or extrapolation in a regulatory setting. Uncertainties that may be of academic interest but would have little impact on regulatory application don't need to be described. In general, this section details evidence that may raise questions regarding the overall validity and predictive utility of the KER (including consideration of both biological plausibility and empirical support). It also contributes along with several other elements to the overall evaluation of the WoE for the KER (see, Section 4).

\section{Quantitative Understanding}

The quantitative understanding section of the KER description is intended to capture information that helps to define how much change in the upstream KE, and/or for how long, is needed to elicit a detectable and defined change in the downstream KE. While empirical support (F) addresses whether data between the two KEs are consistent with the patterns that are expected if the upstream event is causing the downstream event to occur, the quantitative understanding section helps to define the precision with which the state of the downstream KE can be predicted from knowledge of the state of the upstream KE. These quantitative relationships may be defined in terms of correlations, responseresponse relationships, dose-dependent transitions or points of departure (i.e., a threshold of change in KEupstream needed to elicit a change in KEdownstream), etc. They may take the form of simple mathematical equations or sophisticated biologically-based computational models that consider other modulating factors such as compensatory responses, or interactions with other biological or environmental variables. Regardless of form, the idea is to briefly describe what is known regarding the quantitative relationship between the KEs and cite appropriate literature that defines those relationships and/or provides support for them.

Data that confers quantitative understanding of a KER are not necessarily mutually exclusive from those addressing other weight of evidence considersations. In that respect, the quantitative understanding section of the KER description is not intended to be redundant with the other WoE sections. Rather, it is intended to aid application of the AOP by allowing a reader to rapidly identify the relationships that would support quantitative prediction of the probability or magnitude of change in KEdownstream based on a known state of KEupstream. For transparency, the toxicological and biological 
context in which the quantitative relationships were defined should be indicated within the description. However, the ultimate goal is to identify quantitative relationships that generalise across the entire applicability domain of the two KEs being linked via the KER.

Implementation in the AOP-Wiki: https://aopwiki.org/info_pages/2/info_linked_pages/2\#G Quantitative Understanding of the KER

Based on recommendations from workshops held in September 2015 (Wittwehr et al. 2016) and April 2017 (LaLone et al. 2017), description of the quantitative understanding of the KER has been organised into subsections in order to more consistently capture information that would be informative for both quantitative AOP and AOP network applications. As with other areas of the AOP descriptions, authors are encouraged to complete the sections to the extent that is feasible, but it is recognised that supporting information may not be adequate to address all sections.

\section{Response-response relationship}

This subsection should be used to define sources of data that define the response-response relationships between the KEs. In particular, information regarding the general form of the relationship (e.g., linear, exponential, sigmoidal, threshold, etc.) should be captured if possible. If there are specific mathematical functions or computational models relevant to the KER in question that have been defined, those should also be cited and/or described where possible, along with information concerning the approximate range of certainty with which the state of the KEdownstream can be predicted based on the measured state of the KEupstream (i.e., can it be predicted within a factor of two, or within three orders of magnitude?). For example, a regression equation may reasonably describe the response-response relationship between the two KERs, but that relationship may have only been validated/tested in a single species under steady state exposure conditions. Those types of details would be useful to capture.

\section{Time-scale}

This sub-section should be used to provide information regarding the approximate timescale of the changes in KEdownstream relative to changes in KEupstream (i.e., do effects on KEdownstream lag those on KEupstream by seconds, minutes, hours, or days?). This can be useful information both in terms of modelling the KER, as well as for analysing the critical or dominant paths through an AOP network (e.g., identification of an AO that could kill an organism in a matter of hours will generally be of higher priority than other potential AOs that take weeks or months to develop). Identification of time-scale can also aid the assessment of temporal concordance. For example, for a KER that operates on a time-scale of days, measurement of both KEs after just hours of exposure in a short-term experiment could lead to incorrect conclusions regarding dose-response or temporal 
concordance if the time-scale of the upstream to downstream transition was not considered.

\section{Known modulating factors}

This sub-section presents information regarding modulating factors/variables known to alter the shape of the response-response function that describes the quantitative relationship between the two KEs (for example, an iodine deficient diet causes a significant increase in the slope of the relationship; a particular genotype doubles the sensitivity of KEdownstream to changes in KEupstream). Information on these known modulating factors should be listed in this subsection, along with relevant information regarding the manner in which the modulating factor can be expected to alter the relationship (if known). Note, this section should focus on those modulating factors for which solid evidence supported by relevant data and literature is available. It should NOT list all possible/plausible modulating factors. In this regard, it is useful to bear in mind that many risk assessments conducted through conventional apical guideline testing-based approaches generally consider few if any modulating factors.

\section{Known Feedback loops influencing this KER}

This subsection should define whether there are known positive or negative feedback mechanisms involved and what is understood about their time-course and homeostatic limits? In some cases where feedback processes are measurable and causally linked to the outcome, they should be represented as KEs (see development tip 4). However, in most cases these features are expected to predominantly influence the shape of the responseresponse, time-course, behaviours between selected KEs. For example, if a feedback loop acts as compensatory mechanism that aims to restore homeostasis following initial perturbation of a KE, the feedback loop will directly shape the response-response relationship between the KERs. Given interest in formally identifying these positive or negative feedback, it is recommended that a graphical annotation indicating a positive or negative feedback loop (Figure 4) is involved in a particular upstream to downstream KE transition (KER) be added to the graphical representation, and that details be provided in this subsection of the KER description.

A.

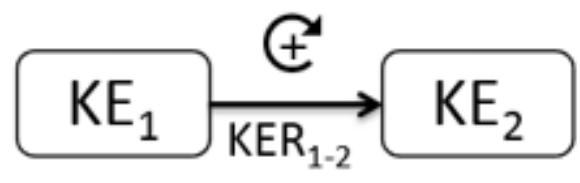

B.

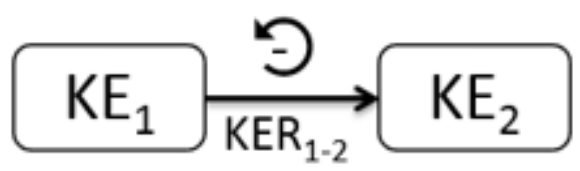

Figure 4. Recommended graphical annotation to indicate that a known (A) positive feedback (i.e., feedforward) or (B) negative feedback loop is involved in the transition from one KE to the next in the AOP. Note: This is an optional annotation. See Section 6D for 
more information on describing positive and negative feedback processes using the AOP framework.

\section{Development tip 5 - Capturing information on positive or negative feedback loops.}

Ways to capture/represent known positive or negative feedback loops have emerged as a frequently asked question in relation to use of the AOP framework. Thus, a few general guidelines are provided here.

- In cases where feedback loops play a direct causal role in the progression of a biological perturbation leading to an AO, they can be included as KEs as long as they are measurable. For example, for an AOP in which a negative feedback process results in decreased hormone signalling that leads to the AO, a measurable event indicative of or involved in the activation of the negative feedback could be included as a KE.

- In cases where a feedback loop may act as a key compensatory or adaptive mechanism that dictates how severely the KEupstream needs to be impacted in order of affect the KEdownstream, but does not play a direct causal role in the AOP (other than defining the relevant point of departure), the feedback should not be included as a separate KE. Rather it should be detailed as part of the quantitative understanding section of the KER description. In the user supplied graphical representation, a forward or backward looping symbol could be added above the arrow linking the two KEs to indicate that a known positive or negative feedback loop is involved in the transition (Figure 4B).

- In cases where two measurable KEs in an AOP are part of a positive feedback loop, it can be challenging to define which should be upstream and which downstream, as they are amplifying or altering one another in a cycle. A two headed arrow is undesirable as it can incorrectly suggest that the AOP is reversible. However, in practice an AOP with a positive feedback loop could be accurately represented as two different AOPs in the AOP-Wiki, in which the KEs involved in the positive feedback are presented in either order. This effectively creates a bi-directional arrow when the AOP network is assembled. Rather than creating two nearly identical AOP pages with the KE order reversed for each, the current recommendation is to select either order for the KEs and connect them with a unidirectional arrow, but add a forward looping symbol above the arrow in the user-supplied graphical representation to indicate that a known feedforward loop is involved (Figure 4A).

\section{Classification of quantitative understanding}

To aid in overall assessment of the AOP and whether it is fit-for-purpose for various applications, developers are also asked to classify the extent of quantitative understanding of the KER as low, moderate, or high. General guidance for classification of the level of 
quantitative understanding of a KER as low, moderate, or high (Annex 2) is based on several key considerations:

- The extent to which a change in KEdownstream can be precisely predicted based on KEupstream.

- The precision with which uncertainty in the prediction of KEdownstream can be quantified.

- The extent to which known modulating factors or feedback mechanisms are accounted for.

- The extent to which the relationships described can be reliably generalised across the biological applicability domain of the KER.

\section{References}

List of the literature that was cited for this KER description using the appropriate format. 
A. Example of acceptable branching on an AOP page. Represents additive/joint ("and" relationships) actions of a single MIE that contribute to a single $\mathrm{AO}$.

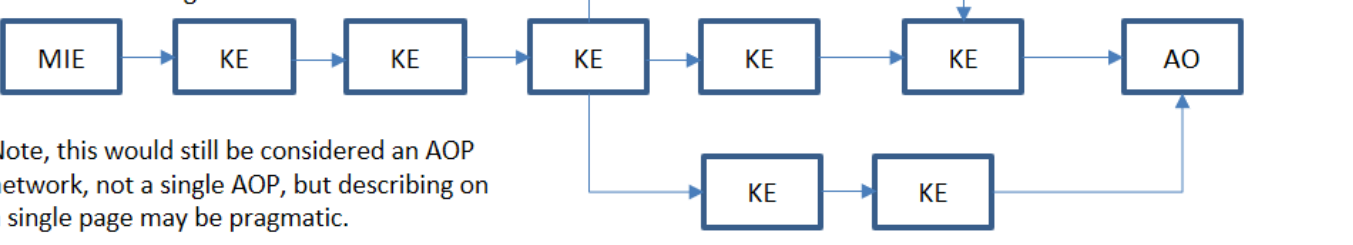

B.

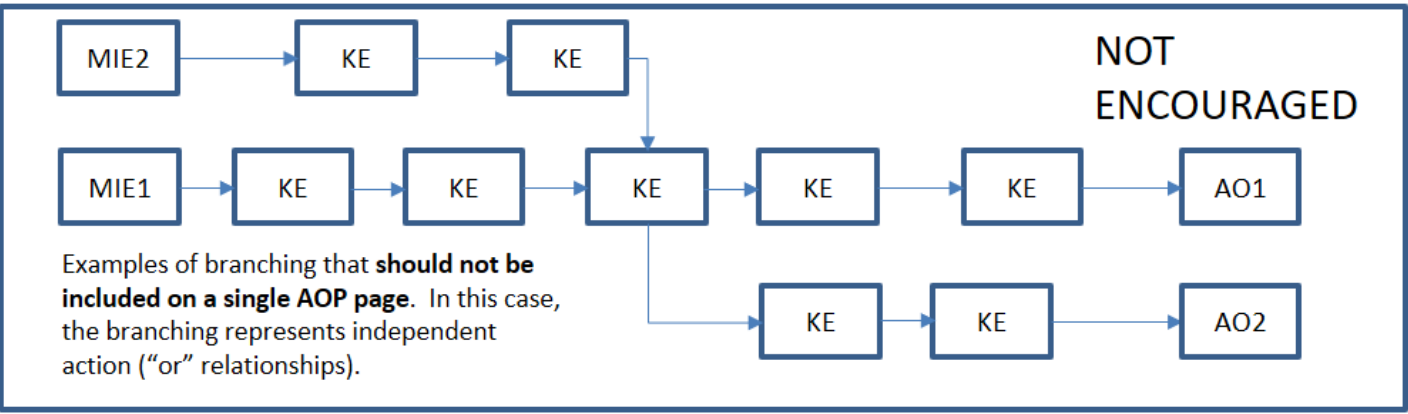

C.

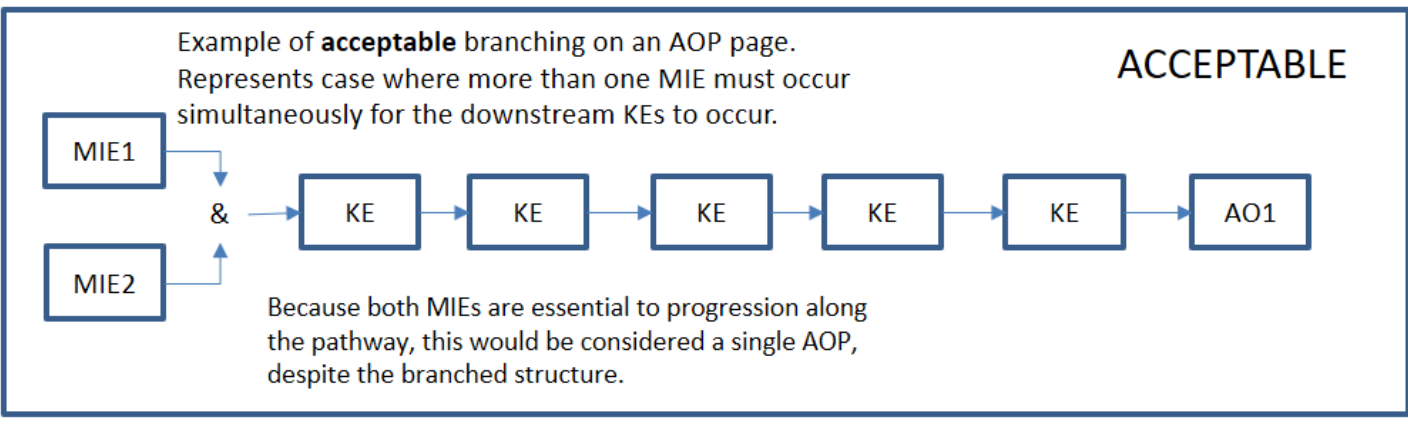

Figure 5. Illustration of general guidance regarding inclusion of simple AOP networks or branched AOP structures (A) on a single AOP page. Branching representing independent actions leading to more than AO should not be included in an AOP description (B). Branching indicating multiple KEs (including MIEs) that MUST occur for the pathway to progress downstream should be included in an AOP description.

\section{Development tip 6 - Branching of AOPs captured on a single AOP page}

In general, individual AOPs are defined as a single, non-branching sequence of KEs, linked by KERs that connect a single MIE to an AO (Villeneuve et al. 2014a). In most cases, this is viewed as the most pragmatic unit for development and evaluation of AOP descriptions. Consequently, most AOPs pages should define a single, non-branching, sequence of KEs linked by KERs. However, it is recognised that in some cases there may be exceptions for which representation of a simple AOP network on an AOP page is a more pragmatic unit of development and evaluation (see Leist et al. 2017 for examples and further explanation). Under certain circumstances, representation of a branched structure on an AOP page is 
acceptable, so long as the principles of modularity of the KEs and KERs and overall coherence to the framework is maintained.

For example, representation of branching on an AOP may become pragmatic when there are multiple KEs, causally linked to the MIE and AO that are occurring concurrently and likely acting in concert to drive the downstream effects. In such cases, the various KEs cannot necessarily be placed neatly into a single temporal sequence because they are effectively occurring simultaneously. Likewise it cannot necessarily be determined which of the concurrent KEs is most essential or critical, because there are multiple KEs (measurable biological changes) contributing jointly in an additive manner such that it cannot be effectively determined whether one could cause the pathway to progress without the other. This is contrasted with cases where KEs act independently such that one event or the other, alone, would allow progression toward the outcome.

In cases where an additive (and) relationship must be assumed, representation of a simple AOP network on a single AOP page within the AOP-KB may be more practical from both a development and use stand-point than breaking those multiple highly related pathways into separate AOP descriptions. As long as KEs and associated KERs are each represented as separate modular pages in the AOP$\mathrm{KB}$ (as described below), capturing such networks on single AOP pages does not create problems for modular AOP network building. Indeed, it can actually strengthen the overall AOP by capturing the evidence for pleiotropic effects of the same MIE that ultimately contribute to the same outcome.

Note, such branched AOP structures should only be included on a single AOP page when all the branches diverge from a common MIE (or MIEs in the case that two or more MIEs MUST occur to drive the pathway) and converge to a common $\mathrm{AO}$ (Figure 5A) and two or more of the KEs contributing causally to the AO occur concurrently such that it is experimentally intractable to isolate and identify which is playing the dominant causal role (i.e., in all likelihood both KEs are contributing) and both (all KEs) measurements are deemed to have predictive value.

Branched structures should not be included on a single AOP page when they diverge to independent outcomes (e.g., Figure 5B) and/or are operating largely independent of one another and can be resolved from one another in space or time, experimentally. Following this logic, two or more MIEs may occur on an AOP page, when more than one event MUST happen simultaneously in order for the pathway to be triggered (Figure 5C). 


\section{Section 4 - Overall Assessment of the AOP}

This section addresses the relevant biological domain of applicability (i.e., in terms of taxa, sex, life stage, etc.) and WoE for the overall AOP as a basis to consider appropriate regulatory application (e.g., priority setting, testing strategies or risk assessment). The goal of the overall assessment is not to reproduce or reiterate all the content assembled as part of sections 1-3, but rather to provide a high level synthesis and overview of the relative confidence in the AOP and where the significant gaps or weaknesses are (if they exist). Users or readers can drill down into the finer details captured in the KE and KER descriptions, and/or associated summary tables, as appropriate to their needs.

Determination of confidence in the overall AOP is based on the biological plausibility, empirical support, and extent of quantitative understanding for the KERs (Section 3) and the evidence supporting essentiality of the KEs.

Assessment of the AOP is organised into a number of steps. Guiding questions that inform evaluation at each step are included in Annexes 1 and 2. The questions are designed to facilitate assignment of categories of high, moderate, or low confidence for each consideration. While it is not necessary to repeat lengthy text that appears elsewhere in the AOP description (or related KE and KER descriptions), a brief explanation or rationale for the selection of high, moderate, or low confidence should be made, in light of the guiding questions detailed below.

Implementation in the AOP-Wiki:

https://aopwiki.org/info_pages/2/info_linked_pages/1\#J Overall Assessment of the AOP

\section{Define the Biological Domain of Applicability of the AOP}

The relevant biological domain(s) of applicability in terms of sex, life-stage, taxa, and other aspects of biological context are defined in this section. Biological domain of applicability is informed by the "Description" and "Biological Domain of Applicability" sections of each KE and KER description (see sections $2 \mathrm{G}$ and $3 \mathrm{E}$ for details). In essence the taxa/life-stage/sex applicability is defined based on the groups of organisms for which the measurements represented by the KEs can feasibly be measured and the functional and regulatory relationships represented by the KERs are operative.

The relevant biological domain of applicability of the AOP as a whole will nearly always be defined based on the most narrowly restricted of its KEs and KERs. For example, if 
most of the KEs apply to either sex, but one is relevant to females only, the biological domain of applicability of the AOP as a whole would be limited to females. While much of the detail defining the domain of applicability may be found in the individual KE and KER descriptions, the rationale for defining the relevant biological domain of applicability of the overall AOP should be briefly summarised on the AOP page.

Implementation in the AOP-Wiki:

https://aopwiki.org/info_pages/2/info_linked_pages/1\#Biological Domain of Applicability

\section{Assess the Essentiality of All KEs}

An important aspect of assessing an AOP is evaluating the essentiality of its KEs. The essentiality of KEs can only be assessed relative to the impact of manipulation of a given $\mathrm{KE}$ (e.g., experimentally blocking or exacerbating the event) on the downstream sequence of KEs defined for the AOP. Consequently evidence supporting essentiality is assembled on the AOP page, rather than on the independent KE pages that are meant to stand-alone as modular units without reference to other KEs in the sequence.

The nature of experimental evidence that is relevant to assessing essentiality relates to the impact on downstream KEs and the AO if upstream KEs are prevented or modified. This includes:

- Direct evidence: directly measured experimental support that blocking or preventing a KE prevents or impacts downstream KEs in the pathway in the expected fashion.

- Indirect evidence: evidence that modulation or attenuation in the magnitude of impact on a specific KE (increased effect or decreased effect) is associated with corresponding changes (increases or decreases) in the magnitude or frequency of one or more downstream KEs.

When assembling the support for essentiality of the KEs, authors should organise relevant data in a tabular format (e.g., Table 5). The objective is to summarise briefly the nature and numbers of investigations in which the essentiality of KEs has been experimentally explored either directly or indirectly. In some cases, the impact of blocking or modifying an early KE on all downstream KEs in the pathway has been determined; in other cases, the impact only on a single adjacent or non-adjacent downstream KE has been measured.

When assembling support for essentiality of the KEs, it is not necessary to repeat lengthy text on the design or results of relevant investigations that may appear in other parts of the AOP description (e.g., as WoE for a KER). Rather, the entries should briefly address the extent of the supporting and contradictory data through a short description of the nature of the direct or indirect evidence addressing essentiality, along with relevant references. The objective is to provide an overview of the extent and nature of supporting 
and inconsistent data on essentiality of the KEs in a format that will facilitate a "call" on the overall degree of support for essentiality across the AOP (Section 8). Some examples of brief narratives addressing support for essentiality are included here. See https://aopwiki.org/info pages/2/info linked pages/6 for additional examples:

For direct evidence:

- Knock-out of KE1 or early KEs leads to blockage of all downstream KEs

- One or more downstream KEs is blocked or reversed by inhibiting (or allowing recovery of) upstream KEs

- Overexpression in repair enzyme for early KEs leads to decreased incidence of downstream KEs

- Antagonism or agonism of upstream KE leads to expected pattern of effects on downstream KEs

For indirect evidence:

- Impact on a modulating factor for early KEs leads to expected pattern of effects on later KEs

Table 5. Example of a Table Format for Assembling the Data on Essentiality of KEs:

\begin{tabular}{|c|c|c|c|c|}
\hline Event & $\begin{array}{l}\text { Direct } \\
\text { Evidence }\end{array}$ & $\begin{array}{l}\text { Indirect } \\
\text { Evidence }\end{array}$ & $\begin{array}{l}\text { No experimental } \\
\text { evidence }\end{array}$ & $\begin{array}{l}\text { Contradictory } \\
\text { experimental } \\
\text { evidence }\end{array}$ \\
\hline \multicolumn{5}{|l|}{ MIE } \\
\hline \multicolumn{5}{|l|}{ KE1 } \\
\hline \multicolumn{5}{|l|}{ KE2 } \\
\hline $\begin{array}{l}\text { KE3 } . . . . . . . \\
\text { KEn }\end{array}$ & & & & \\
\hline
\end{tabular}

Where there is no experimental model to prevent or augment a specific KE in the context of the overall pathway, this should be indicated as "No data".

\section{Uncertainties or Inconsistencies:}

In addition to outlining the evidence supporting essentiality, it is also important to identify inconsistencies or uncertainties, as presented in one of the columns in Table 5. This could include, for example, evidence in specific studies that did not support that blockage or attenuation of an early KE impacted later KEs in the AOP. Discordance with the results of other studies should be considered based on evaluation of the adequacy of study design, taking into account, for example, the sensitivity of the detection of impact. It could also include, for example, gaps in knowledge concerning the essentiality of the MIE or particular KEs where there are data on essentiality only for one or a few. To the 
extent possible, inconsistencies and uncertainties should focus on data gaps important for potential envisaged regulatory applications as a basis for indicating priorities for further research.

Based on the assembled evidence on essentiality for the KEs, confidence in the supporting data on essentiality is considered for the entire AOP, including KERs and KEs. This is commonly based on the extent of direct and/or indirect evidence for one, several or all of the KEs.

Confidence in the supporting data for essentiality of KEs within the AOP is considered:

- $\quad$ High if there is direct evidence from specifically designed experimental studies illustrating prevention or corresponding impact on downstream KEs and/or the AO if upstream KEs are blocked or modified (e.g., via stop exposure/reversibility studies, antagonism, knock out models, etc.);

- Moderate if there is indirect evidence that modification of one or more upstream KEs is associated with a corresponding (increase or decrease) in the magnitude or frequency of downstream KEs [e.g., augmentation of proliferative response (KEupstream) leading to increase in tumour formation (KEdownstream or AO)];

- Low if there is no or contradictory experimental evidence that blocking or modulating/attenuating any of the KEs influences the KEs downstream or AO (Annex 1).

These considerations, as well as those related to biological plausibility and empirical support draw upon experience in application of WoE analysis in mode of action analysis in chemical specific regulatory application, an important envisaged application of AOPs. As such, they reflect broad collective and evolving experience in regulatory application of mechanistic data, tailored to maintain balance between relevant aspects of application with envisaged modular development of AOPs. For essentiality, considerations also reflect the nature of experimental data that optimally informs this critical component for regulatory application. Supporting experimental investigations which address the impact of early key events on all subsequent KEs in hypothesized AOPs obviate the need for studies on the essentiality of individual KEs.

Implementation in the AOP-Wiki:

https://aopwiki.org/info_pages/2/info_linked_pages/1\#Essentiality of the Key Events

\section{Assess the Evidence Supporting All KERs}

The biological plausibility, empirical support, and quantitative understanding from each KER in an AOP are assessed together: 


\section{Review the Biological Plausibility of Each KER}

Biological plausibility of each of the KERs in the AOP is the most influential consideration in assessing $\mathrm{WoE}$ or degree of confidence in an overall hypothesised AOP for potential regulatory application (Meek et al., 2014; 2014a). The defining question for biological plausibility (Annex 1) is: Is there a mechanistic (i.e., structural or functional) relationship between KEupstream and KEdownstream consistent with established biological knowledge? WoE for the biological plausibility of the KERs would be considered:

- $\quad$ High if it is well understood based on extensive previous documentation and has an established mechanistic basis and broad acceptance (e.g., increased follicle stimulating hormone signalling leading to increased estrogen synthesis, increased incidence of alkylated DNA leading to increased incidence of mutations);

- Moderate if the KER is plausible based on analogy to accepted biological relationships but scientific understanding is not completely established;

- Low if there is empirical support for a statistical association between KEs but structural or functional relationship between them is not understood.

\section{Review the Empirical Support for Each KER}

Empirical support entails consideration of experimental data in terms of the associations between KEs - namely dose-response concordance and temporal relationships between and across multiple KEs. It is examined most often in studies of dose-response/incidence and temporal relationships for stressors that impact the pathway. While less influential than biological plausibility of the KERs and essentiality of the KEs (Meek et al., 2014; 2014a), empirical support can increase confidence in the relationships included in an AOP.

It is important to recognise that empirical support relates to the "concordance" of dose response, temporal and incidence relationships for KERs; the defining question is not whether or not there is a dose response relationship for a specific KE but rather, whether there is expected concordance with the dose-response relationships for KERs - i.e., between KEs.

The defining questions for empirical support (Annex 1) are: Does KEupstream occur at lower doses and earlier time points than KEdownstream; is the incidence or frequency of KEupstream greater than that for KEdownstream for the same dose of tested stressor? Inconsistencies in empirical support across taxa, species and stressors that don't align with the expected pattern for the hypothesised AOP as described in Section 3 should be identified and their basis considered.

Empirical support for each of the KERs would be considered:

- $\underline{\text { High }}$ if there is dependent change in both events following exposure to a wide range of specific stressors (extensive evidence for temporal, dose-response and incidence concordance) and no or few data gaps or conflicting data; 
- Moderate if there is demonstrated dependent change in both events following exposure to a small number of specific stressors and some evidence inconsistent with the expected pattern that can be explained by factors such as experimental design, technical considerations, differences among laboratories, etc.;

- Low if there are limited or no studies reporting dependent change in both events following exposure to a specific stressor (i.e., endpoints never measured in the same study or not at all), and/or lacking evidence of temporal or dose-response concordance, or identification of significant inconsistencies in empirical support across taxa and species that don't align with the expected pattern for the hypothesised AOP.

Tables summarising the relevant experimental data for tested stressors may be helpful in considering the extent of empirical support. For example, benchmark doses (BMDs) for specified similar increases in of each of the KEs are entered in the cells of the table. If the hypothesised linkages in the AOP are supported by empirical data, there is a pattern of increasing BMDs from the top left hand corner to the bottom right hand corner for each of the tested stressors. Presentation in this manner readily identifies any exceptions to the expected patterns that are considered as inconsistencies and diminish from the overall weight of empirical support (see Tables 6).

Table 6. Generic example of a concordance table for evaluating empirical support of the KERs.

Type the subtitle here. If you do not need a subtitle, please delete this line.

\begin{tabular}{llllllll}
\hline Species tested & Stressor & Time pt & KE1 $^{\mathbf{a}}$ & KE2 & KE3 & KE4 & KE5 \\
\hline FHM & A & $6 \mathrm{~h}$ & 1 & No effect & No effect & No data & No effect \\
FHM & A & $24 \mathrm{~h}$ & 1 & 10 & No effect & No data & No effect \\
FHM & A & $4 \mathrm{~d}$ & No effect & 1 & 10 & 20 & 50 \\
FHM & $\mathrm{A}$ & $8 \mathrm{~d}$ & No effect & 1 & 10 & 10 & 20 \\
FHM & $\mathrm{A}$ & $21 \mathrm{~d}$ & No effect & 1 & 10 & 10 & 10 \\
FHM & $\mathrm{B}$ & $24 \mathrm{~h}$ & 25 & 25 & 50 & No data & No data \\
FHM & $\mathrm{B}$ & $10 \mathrm{~d}$ & 10 & 10 & 25 & 25 & 25 \\
RBT & $\mathrm{A}$ & $12 \mathrm{~h}$ & 0.2 & 0.2 & 10 & 10 & No effect \\
RBT & $\mathrm{A}$ & $24 \mathrm{~h}$ & 0.2 & 0.2 & 1 & 10 & 10 \\
RBT & $\mathrm{A}$ & $8 \mathrm{~d}$ & 0.1 & 0.2 & 0.5 & 0.5 & 0.5 \\
RBT & $\mathrm{A}$ & $21 \mathrm{~d}$ & 0.1 & 0.1 & 0.2 & 0.5 & 0.5 \\
\hline
\end{tabular}

a: Benchmark dose at which a specified level of change in the KE relative to controls was inferred, based on the empirical results. (Note, where concentrations tested are inadequate to determine a BMD, LOEC or NOEC could also be considered, but concentrations tested in different studies must be taken into account).

Additional examples of concordance tables:

- https://aopwiki.org/wiki/images/4/45/Aromatase_inhibition_doseresponse_concordance_table_rev1.pdf 
- https://aopwiki.org/system/dragonfly/production/2017/05/19/1qoq9ky7zb_AOP15 supporting evidence.pdf

- https://aopwiki.org/wiki/index.php/File:Mechanistic data matrix.jpg

- https://aopwiki.org/system/dragonfly/production/2017/03/20/lk2chhiib_AR_agoni sm concordance table updated 2017 03 14.pdf

Implementation in the AOP-Wiki:

https://aopwiki.org/info_pages/2/info_linked_pages/1\#Evidence Assessment

\section{Quantitative WoE considerations (optional)}

Some proof of concept examples to address the WoE considerations for AOPs quantitatively have recently been developed, based on the rank ordering of the relevant Bradford Hill considerations (i.e., biological plausibility, essentiality and empirical support) (Becker et al., 2017; Becker et al, 2015; Collier et al., 2016). Suggested quantitation of the various elements is expert derived, without collective consideration currently of appropriate reporting templates or formal expert engagement. Though not essential, developers may wish to assign comparative quantitative values to the extent of the supporting data based on the three critical Bradford Hill considerations for AOPs, as a basis to contribute to collective experience.

\section{Review the Quantitative Understanding for Each KER}

The extent of quantitative understanding of the KERs in an AOP is also critical in consideration with regard to potential regulatory application. For some applications (e.g., dose- response analysis in in-depth risk assessment), quantitative characterisation of downstream KERs may be essential, while for others quantitative understanding of upstream KERs may be most important (e.g., QSAR modelling for category formation for testing). Because evidence that contributes to quantitative understanding of the KER is generally not mutually exclusive with the empirical support for the KER (i.e., expected patterns of quantitative relationships), evidence that contributes to quantitative understanding will generally be considered to some extent as part of the evaluation of the WoE supporting the KER (see Section 3.E. and Annex 1, footnote b). However, specific attention is also given to how precisely and accurately one can potentially predict an impact on KEdownstream based on some measurement of KEupstream. This is captured in the form of quantitative understanding calls for each KER. As noted in section 3, general guidance for characterising the level of quantitative understanding of a KER as low, moderate, or high (Annex 2) is based on several key considerations:

- The extent to which a change in KEdownstream can be precisely predicted based on KEupstream.

- The precision with which uncertainty in the prediction of KEdownstream can be quantified.

- The extent to which known modulating factors or feedback mechanisms are accounted for. 
- The extent to which the relationships described can be reliably generalized across the applicability domain of the KER.

As with the other parts of the overall assessment of the AOP, it is not necessary to repeat all the details provided in the KER descriptions. The overall evaluation of the quantitative understanding should briefly explain the rationale for the assigned level of quantitative understanding of each KER.

Implementation in the AOP-Wiki:

https://aopwiki.org/info_pages/2/info_linked_pages/1\#Quantitative Considerations 


\section{References}

Becker RA, Ankley GT, Edwards SW, Kennedy SW, Linkov I, Meek B, Sachana M, Segner H, Van Der Burg B, Villeneuve DL, Watanabe H, Barton-Maclaren TS. (2015). Increasing scientific confidence in adverse outcome pathways: application of tailored Bradford-Hill considerations for evaluating weight of evidence. Regul Toxicol Pharmacol 72: 514-537.

Becker RA, Dellarco V, Seed J, Kronenberg JM, Meek B, Foreman J, Palermo C, Kirman C, Linkov I, Schoeny R, Dourson M. (2017). Quantitative weight of evidence to assess confidence in potential modes of action. Regul Toxicol Pharmacol 86: 205-220.

Collier ZA, Gust KA, Gonzalez-Morales B, Gong P, Wilbanks MS, Linkov I, Perkins EJ. (2016). A weight of evidence assessment approach for adverse outcome pathways. Regul Toxicol Pharmacol 75: 46-57.

Knapen, D., Vergauwen, L., Villeneuve, D.L. and Ankley GT. (2015) The potential of AOP networks for reproductive and developmental toxicity assay development. Reprod Toxicol. 56: 52-55.

Krewski D, Acosta D Jr., Andersen M, Anderson H, Bailar J.C. 3rd, Boekelheide K, Brent R, Charnley G, Cheung VG, Green S Jr, Kelsey KT, Kerkvliet NI, Li AA, McCray L, Meyer O, Patterson RD, Pennie W, Scala RA, Solomon GM, Stephens M, Yager J, Zeise L. (2010). Toxicity testing in the 21st century: a vision and strategy. J Toxicol Environ Health B Crit Rev. 13: 51-138.

LaLone CA, Ankley GT, Belanger SE, Embry MR, Hodges G, Knapen D, Munn S, Perkins EJ, Rudd MA, Villeneuve DL, Whelan M, Willett C, Zhang X, Hecker M. (2017.) Advancing the adverse outcome pathway framework - an international horizon scanning approach. Environ Toxicol Chem. (in press).

Leist M, Ghallab A, Graepel R, Marchan R, Hassan R, Bennekou SH, Limonciel A, Vinken M, Schildknecht S, Waldmann T, Danen E, van Ravenzwaay B, Kamp H, Gardner I, Godoy P, Bois FY, Braeuning A, Reif R, Oesch F, Drasdo D, Höhme S, Schwarz M, Hartung T, Braunbeck T, Beltman J, Vrieling H, Sanz F, Forsby A, Gadaleta D, Fisher C, Kelm J, Fluri D, Ecker G, Zdrazil B, Terron A, Jennings P, van der Burg B, Dooley S, Meijer AH, Willighagen E, Martens M, Evelo C, Mombelli E, Taboureau O, 
Mantovani A, Hardy B, Koch B, Escher S, van Thriel C, Cadenas C, Kroese D, van de Water B, Hengstler JG. (2017) Adverse outcome pathways: opportunities, limitations, and open questions. Regulat. Toxicol. DOI: 10.1007/s00204-017-2045-3

Meek ME, Klaunig JE. (2010). Proposed mode of action of benzene induced leukemia: interpreting available data and identifying critical data gaps for risk assessment. Chem. Biol. Interact. 184: 279-285.

Meek ME, Boobis AR, Cote I, Dellarco V, Fotakis G, Munn S, Seed J, Vickers C. (2014a). New developments in the evolution and application of the WHO/IPCS framework on mode of action/species concordance analysis. J Appl Toxicol. 34: 1-18.

Meek ME, Palermo CM, Bachman AN, North, CM, Lewis RJ. (2014b). Mode of Action Human Relevance (MOA/HR) Framework - Evolution of the Bradford Hill Considerations and Comparative Analysis of Weight of Evidence. J Appl Toxicol. 34: 595-606.

OECD (2015), OECD Style Guide third edition, OECD Publishing, Paris. https://www.oecd.org/about/publishing/OECD-Style-Guide-Third-Edition.pdf

Villeneuve DL, Crump D, Garcia-Reyero N, Hecker M, Hutchinson TH, LaLone CA, Landesmann B, Lettieri T, Munn S, Nepelska M, Ottinger MA, Vergauwen L, Whelan M. (2014a) Adverse outcome pathway (AOP) development I: strategies and principles. Toxicol Sci. 142: 312-320.

Villeneuve DL, Crump D, Garcia-Reyero N, Hecker M, Hutchinson TH, LaLone CA, Landesmann B, Lettieri T, Munn S, Nepelska M, Ottinger MA, Vergauwen L, Whelan M. (2014b) Adverse outcome pathway development II: best practices. Toxicol Sci. 142: 321 330.

Wittwehr C, Aladjov H, Ankley G, Byrne HJ, de Knecht J, Heinzle E, Klambauer G, Landesmann B, Luijten M, MacKay C, Maxwell G, Meek ME, Paini A, Perkins E, Sobanski T, Villeneuve D, Waters KM, Whelan M. (2017) How Adverse Outcome Pathways can aid the development and use of computational prediction models for regulatory toxicology. Toxicol Sci. 155: 326-336. 


\section{Annex 1: Guidance for Assessing Relative Level of Confidence in the Overall AOP}

Examples of complete tables for selected AOPs are available:

\begin{tabular}{|c|c|}
\hline $\mathrm{AOP}$ & Assessment Summary File \\
\hline https://aopwiki.o & https://aopwiki.org/system/dragonfly/production/2017/05/19/7s1ibrunwt_RevisedAss \\
\hline rg/aops/15 & essmentSummaryAop 15.pdf \\
\hline https://aopwiki.o & https://aopwiki.org/system/dragonfly/production/2017/03/20/3usvv7naq8_Annex1_fo \\
\hline rg/aops/23 & r AOP 23 AR reproductive dys 201703 20.pdf \\
\hline$\frac{\text { https://aopwiki.o }}{\text { rg/aops/38 }}$ & https://aopwiki.org/aops/38\#evidence \\
\hline$\underline{\text { https://aopwiki.o }}$ & https://aopwiki.org/system/dragonfly/production/2017/03/24/6u60jhkjp8_TPO_AOP \\
\hline $\mathrm{rg} / \mathrm{aops} / 42$ & Summary Tables.pdf \\
\hline
\end{tabular}

\begin{tabular}{|c|c|c|c|c|}
\hline & Defining Question & $\mathrm{High}^{2}$, & Moderate & Low \\
\hline $\begin{array}{l}\text { 1. Support for } \\
\text { Biological } \\
\text { Plausibility of } \\
\text { KERs }^{1}\end{array}$ & $\begin{array}{l}\text { Is there a mechanistic } \\
\text { (i.e., structural or } \\
\text { functional) } \\
\text { relationship between } \\
\text { KEup and KEdown } \\
\text { consistent with } \\
\text { established biological } \\
\text { knowledge? }\end{array}$ & $\begin{array}{l}\text { Extensive } \\
\text { understanding } \\
\text { based on extensive } \\
\text { previous } \\
\text { documentation and } \\
\text { broad acceptance } \\
\text {-Established } \\
\text { mechanistic basis }\end{array}$ & $\begin{array}{l}\text { The KER is plausible } \\
\text { based on analogy to } \\
\text { accepted biological } \\
\text { relationships but } \\
\text { scientific } \\
\text { understanding is not } \\
\text { completely } \\
\text { established. }\end{array}$ & $\begin{array}{l}\text { There is empirical } \\
\text { support for a statistical } \\
\text { association between } \\
\text { KEs (See 3.), but the } \\
\text { structural or functional } \\
\text { relationship between } \\
\text { them is not understood. }\end{array}$ \\
\hline $\begin{array}{l}{ }^{4} \mathrm{MIE}=>\mathrm{KE} 1: \\
\text { (copy and } \\
\text { paste the KER } \\
\text { description } \\
\text { into this cell) }\end{array}$ & \multicolumn{4}{|c|}{$\begin{array}{l}\text { Biological Plausibility of the } \mathrm{MIE}=>\mathrm{KE} 1 \text { is } \mathrm{xxx} \text {. } \\
\text { Rationale: }\end{array}$} \\
\hline
\end{tabular}

\footnotetext{
${ }^{1}$ Rank ordered Bradford Hill considerations adapted from Meek et al. (2014b)

2 The guidance for "high", "moderate" and "low" draws on limited current experience. Additional delineation of the nature of relevant evidence in these broadly defined categories requires more experience with larger numbers of documented AOPs.

3 "Direct evidence" implies specifically designed experiments to consider the relevant element. "Indirect evidence" may overlap with other elements.

${ }^{4}$ To the extent possible, each of the relevant Bradford Hill considerations is addressed for each of the KERs (biological plausibility and empirical support) and KEs (essentiality) and separate rationales provided.
} 


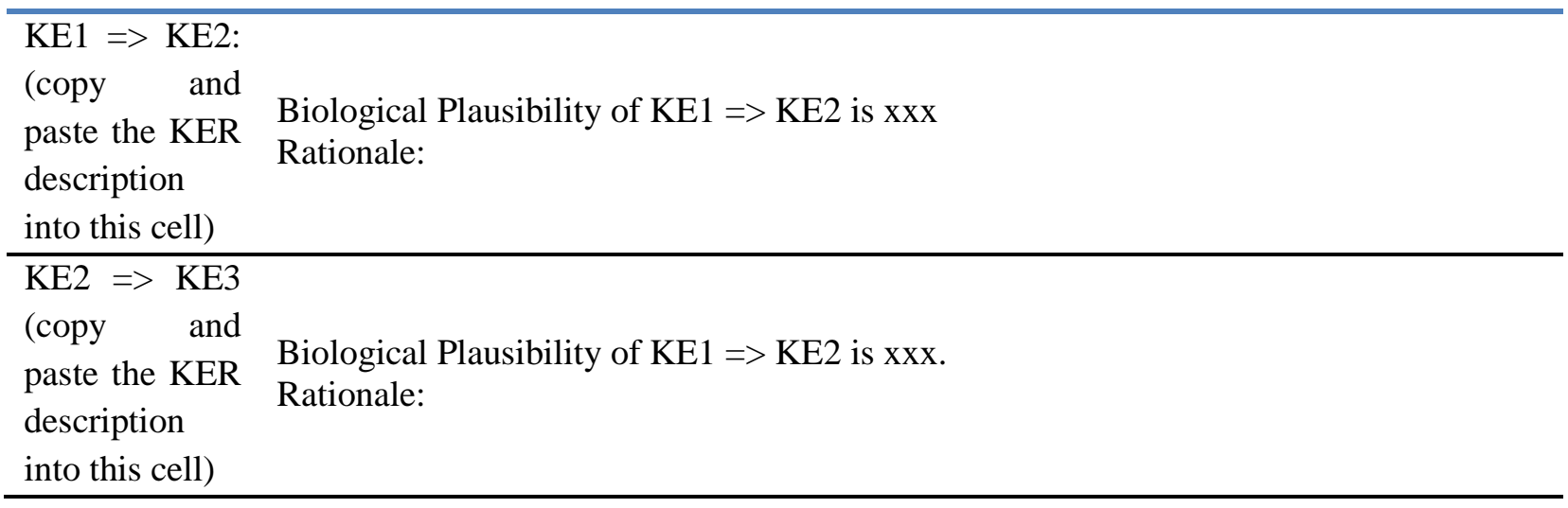

\begin{tabular}{|c|c|c|c|c|}
\hline $\begin{array}{l}\text { 2. Support for } \\
\text { Essentiality of }\end{array}$ & $\begin{array}{l}\text { Defining } \\
\text { Question }\end{array}$ & High & Moderate & Low \\
\hline $\mathrm{KEs}^{5}$ & $\begin{array}{l}\text { What is the } \\
\text { impact on } \\
\text { downstream } \\
\text { KEs and/or the } \\
\text { AO if an } \\
\text { upstream KE } \\
\text { is modified or } \\
\text { prevented? }\end{array}$ & $\begin{array}{l}\text { Direct evidence from } \\
\text { specifically designed } \\
\text { experimental studies } \\
\text { illustrating prevention } \\
\text { or impact on } \\
\text { downstream KEs } \\
\text { and/or the AO if } \\
\text { upstream KEs are } \\
\text { blocked or modified }\end{array}$ & $\begin{array}{l}\text { Indirect evidence that } \\
\text { modification of one or } \\
\text { more upstream KEs is } \\
\text { associated with a } \\
\text { corresponding } \\
\text { (increase or decrease) } \\
\text { in the magnitude or } \\
\text { frequency of } \\
\text { downstream KEs }\end{array}$ & $\begin{array}{l}\text { No or contradictory } \\
\text { experimental evidence of } \\
\text { the essentiality of any of } \\
\text { the KEs. }\end{array}$ \\
\hline $\mathrm{AOP}$ & \multicolumn{4}{|c|}{ Rationale for Essentiality of KEs in the AOP is $\mathrm{xxx}$ : } \\
\hline
\end{tabular}

\begin{tabular}{|c|c|c|c|c|}
\hline & $\begin{array}{l}\text { Defining } \\
\text { Questions }\end{array}$ & High & Moderate & Low \\
\hline $\begin{array}{l}\text { 3. Empirical } \\
\text { Support }^{\mathrm{a}} \text { for } \\
\text { KERs }\end{array}$ & $\begin{array}{l}\text { Does KEup } \\
\text { occur at lower } \\
\text { doses and } \\
\text { earlier time } \\
\text { points than KE } \\
\text { down and at } \\
\text { the same dose } \\
\text { of stressor, is } \\
\text { the incidence } \\
\text { of KEup > }\end{array}$ & $\begin{array}{l}\text { Multiple studies } \\
\text { showing dependent } \\
\text { change in both events } \\
\text { following exposure to a } \\
\text { wide range of specific } \\
\text { stressors. (Extensive } \\
\text { evidence for temporal, } \\
\text { dose- response and } \\
\text { incidence concordance) } \\
\text { and no or few critical }\end{array}$ & $\begin{array}{l}\text { Demonstrated } \\
\text { dependent change in } \\
\text { both events following } \\
\text { exposure to a small } \\
\text { number of specific } \\
\text { stressors and some } \\
\text { evidence inconsistent } \\
\text { with expected pattern } \\
\text { that can be explained } \\
\text { by factors such as }\end{array}$ & $\begin{array}{l}\text { Limited or no studies } \\
\text { reporting dependent } \\
\text { change in both events } \\
\text { following exposure to a } \\
\text { specific stressor (i.e., } \\
\text { endpoints never measured } \\
\text { in the same study or not at } \\
\text { all); and/or significant } \\
\text { inconsistencies in } \\
\text { empirical support across }\end{array}$ \\
\hline
\end{tabular}

\footnotetext{
${ }^{5}$ While the extent of the supporting data on the essentiality of each of the KEs is addressed separately (Table 5), delineation of the degree of confidence is based on consideration of evidence for all of the KEs within the AOP and therefore, only one rationale is required. This call is normally based on the extent of the available evidence for a range of KEs in the AOP.
} 


\begin{tabular}{|c|c|c|c|}
\hline & $\begin{array}{l}\text { than that for } \\
\text { KEdown? }{ }^{6}, \\
\text { Are there } \\
\text { inconsistencies } \\
\text { in empirical } \\
\text { support } \\
\text { across taxa, } \\
\text { species and } \\
\text { stressors that } \\
\text { don't align } \\
\text { with } \\
\text { expected } \\
\text { pattern for } \\
\text { hypothesised } \\
\text { AOP? }\end{array}$ & $\begin{array}{l}\text { experimental design, } \\
\text { technical } \\
\text { considerations, } \\
\text { differences among } \\
\text { laboratories, etc. }\end{array}$ & $\begin{array}{l}\text { taxa and species that don't } \\
\text { align with expected pattern } \\
\text { for hypothesised AOP }\end{array}$ \\
\hline $\begin{array}{l}\text { MIE => KE1: } \\
\text { (copy and } \\
\text { paste the KER } \\
\text { description } \\
\text { into this cell) }\end{array}$ & \multicolumn{3}{|c|}{ Empirical Support of the MIE $=>\mathrm{KE} 1$ is. $\mathrm{xxx}$. Rationale: } \\
\hline $\begin{array}{l}\text { KE1 => KE2 : } \\
\text { (copy and } \\
\text { paste the KER } \\
\text { description } \\
\text { into this cell) }\end{array}$ & \multicolumn{3}{|c|}{ Empirical Support of the KE1 => KE2 is $\mathrm{xxx}$. Rationale: } \\
\hline $\begin{array}{l}\text { KE2 => KE3 } \\
\text { (copy and } \\
\text { paste the KER } \\
\text { description } \\
\text { into this cell) }\end{array}$ & \multicolumn{3}{|c|}{ Empirical Support of the KE1 $=>$ KE2 is $\mathrm{xxx}$. Rationale: } \\
\hline $\begin{array}{l}\text { a In many cases } \\
\text { also provide em } \\
\text { "Quantitative } \mathrm{U} \\
\text { weight of evide }\end{array}$ & $\begin{array}{l}\text { evidence that contributes to quantitativ } \\
\text { irical support for the relationship. Con } \\
\text { derstanding" section of the KER descr } \\
\text { ce evaluation of the concordance of en }\end{array}$ & $\begin{array}{l}\text { understanding (Sect } \\
\text { quently, relevant in } \\
\text { tion should be consi } \\
\text { irical observations a }\end{array}$ & $\begin{array}{l}\text { 3-KER descriptions) will } \\
\text { lation from the } \\
\text { as part of the overall } \\
\text { onsistency for the KER. }\end{array}$ \\
\hline
\end{tabular}

\footnotetext{
${ }^{6}$ This is normally considered on the basis of tabular presentation of available data on temporal and dose-response aspects, in a template that documents the extent of support. See, for example, Table 6.

${ }^{7}$ Note that this relates to concordance of dose response, temporal and incidence relationships for KERs rather than the KEs; the defining question is not whether or not there is a dose response relationship for the KE but rather there is concordance with that for earlier and later KEs. This is normally demonstrated in studies with different types of stressors.
} 


\section{Annex 2: General guidance for characterising the level of quantitative understanding of a KER as low, moderate, or high.}

\begin{tabular}{ll}
\hline $\begin{array}{l}\text { Extent of } \\
\text { Quantitative } \\
\text { Understanding }\end{array}$ & Characteristics \\
\hline & $\begin{array}{l}\text { Change in KEdownstream can be precisely predicted based on a } \\
\text { relevant measure of KEupstream. } \\
\text { Uncertainty in the quantitative prediction can be precisely } \\
\text { estimated from the variability in the relevant measure of }\end{array}$ \\
KEupstream. \\
Known modulating factors and feedback/feedforward \\
mechanisms are accounted for in the quantitative description. \\
There is evidence that the quantitative relationship between the \\
KEs generalises across the relevant applicability domain of the \\
KER. \\
Change in KEdownstream can be precisely predicted based on a \\
relevant measure of KEupstream. \\
Uncertainty in the quantitative prediction is influenced by factors \\
other than the variability in the relevant measure of KEupstream. \\
Quantitative description does not account for all known \\
modulating factors and/or known feedback/feedforward \\
mechanisms. \\
The quantitative relationship has only been demonstrated for a \\
subset of the overall applicability domain of the KER (e.g., based \\
on a single species). \\
Only a qualitative or semi-quantitative prediction of the change in \\
KEdownstream can be determined from a measure of \\
KEupstream. \\
Known modulating factors and/or known feedback/feedforward \\
mechanisms are not accounted for. \\
The quantitative relationship has only been demonstrated for a \\
narrow subset of the overall applicability domain of the KER \\
(e.g., based on a single species).
\end{tabular}

